\title{
Heat Kernel Estimates of Fractional Schrödinger Operators with Negative Hardy Potential
}

\section{Tomasz Jakubowski ${ }^{1}$ (D) . Jian Wang ${ }^{2}$}

Received: 18 October 2018 / Accepted: 24 July 2019 / Published online: 16 August 2019

(C) The Author(s) 2019

\section{Abstract}

We obtain two-sided estimates for the heat kernel (or the fundamental function) associated with the following fractional Schrödinger operator with negative Hardy potential

$$
\Delta^{\alpha / 2}-\lambda|x|^{-\alpha}
$$

on $\mathbb{R}^{d}$, where $\alpha \in(0, d \wedge 2)$ and $\lambda>0$. The proof is purely analytical and elementary. In particular, for upper bounds of heat kernel we use the Chapman-Kolmogorov equation and adopt self-improving argument.

Keywords Fractional Laplacian · Hardy potential · Heat kernel · The Chapman-Kolmogorov equation · The Feynman-Kac formula · Duhamel's formula

Mathematics Subject Classification (2010) 60G51 · 60G52 - 60J25 · 60J75

\section{Introduction}

Let $d \in \mathbb{N}_{+}:=\{1,2, \cdots\}$ and $\alpha \in(0, d \wedge 2)$. We consider the following Schrödinger operator

$$
\mathcal{L}:=\Delta^{\alpha / 2}+q
$$

Tomasz Jakubowski is partially supported by the NCN grant 2015/18/E/ST1/00239 and by Wrocław University of Science and Technology, grant 0401/0155/18. Jian Wang is partially supported by the NNSFC (No. 11831014), the Program for Probability and Statistics: Theory and Application (No. IRTL1704), and the Program for IRTSTFJ

Tomasz Jakubowski

tomasz.jakubowski@pwr.edu.pl

Jian Wang

jianwang@fjnu.edu.cn

1 Faculty of Pure and Applied Mathematics, Wrocław University of Science and Technology, Wyb. Wyspiańskiego 27, 50-370 Wrocław, Poland

2 College of Mathematics and Informatics \& Fujian Key Laboratory of Mathematical Analysis and Applications (FJKLMAA), Fujian Normal University, 350007 Fuzhou, People's Republic of China 
on $\mathbb{R}^{d}$, where $\Delta^{\alpha / 2}:=-(-\Delta)^{\alpha / 2}$ and $q(x)=\kappa|x|^{-\alpha}$ with

$$
\kappa=\kappa_{\delta}:=\frac{2^{\alpha} \Gamma\left(\frac{\alpha-\delta}{2}\right) \Gamma\left(\frac{d+\delta}{2}\right)}{\Gamma\left(\frac{-\delta}{2}\right) \Gamma\left(\frac{d+\delta-\alpha}{2}\right)}
$$

for any $\delta \in(0, \alpha)$. Here, $\Gamma(-\delta / 2)=\int_{0}^{\infty} r^{-1-\delta / 2}\left(e^{-r}-1\right) d r<0$ for $\delta \in(0, \alpha)$, and $\Gamma(z)=\int_{0}^{\infty} r^{z-1} e^{-r} d r$ for all $z>0$. We note that $\kappa_{\delta}<0$, and so $q(x)<0$ on $\mathbb{R}^{d}$; we also note that the radial function $|x|^{-\alpha}$ comes from the Hardy inequality for fractional Laplacian $\Delta^{\alpha / 2}$ (see [7, 20] and the references therein for more details). Thus, the operator $\mathcal{L}$ given by Eq. 1.1 is the fractional Schrödinger operator with negative Hardy potential. Denote by $\tilde{p}(t, x, y)$ the heat kernel associated with the operator $\mathcal{L}=\Delta^{\alpha / 2}+q$; see Section 2.2 below for more details. Our main result is as follows.

Theorem 1.1 For any $\delta \in(0, \alpha)$, the Schrödinger operator $\mathcal{L}$ given by Eq. 1.1 has the heat kernel $\tilde{p}(t, x, y)$, which is jointly continuous on $(0, \infty) \times \mathbb{R}^{d} \times \mathbb{R}^{d}$, and satisfies two-sided estimates as follows

$$
\tilde{p}(t, x, y) \approx\left(1 \wedge \frac{|x|}{t^{1 / \alpha}}\right)^{\delta}\left(1 \wedge \frac{|y|}{t^{1 / \alpha}}\right)^{\delta}\left(t^{-d / \alpha} \wedge \frac{t}{|x-y|^{d+\alpha}}\right), \quad x, y \in \mathbb{R}^{d}, t>0 .
$$

We note that the last expression in Eq. 1.3 may be replaced by the heat kernel $p(t, x, y)$ of $\Delta^{\alpha / 2}$ (see Section 2.1 and Eq. 2.2). As pointed out before Lemma 2.3 below, the function $\delta \mapsto \kappa_{\delta}$ is strictly decreasing on $(0, \alpha)$ with $\lim _{\delta \rightarrow 0} \kappa_{\delta}=0$ and $\lim _{\delta \rightarrow \alpha} \kappa_{\delta}=-\infty$. Hence, Theorem 1.1 essentially gives us two-sided estimates and the joint continuity of heat kernel associated with the operator $\Delta^{\alpha / 2}-\lambda|x|^{-\alpha}$ for all $\lambda>0$. It is well known that the fractional Laplacian $\Delta^{\alpha / 2}$ is the infinitesimal generator of the rotationally symmetric $\alpha$-stable process, which now has attracted a lot of interests in the field of probability and potential theory (see [6] and references therein). Recently there are also a few works concerning on gradient perturbations and Schrödinger perturbations of fractional Laplacian (see e.g. [9-12, 15, 16, $24,27,28,33,34])$. In particular, according to [33, Theorem 3.4], when the potential belongs to the so-called Kato class, heat kernel estimates for Schrödinger perturbations of fractional Laplacian are comparable with these for fractional Laplacian (at least for any fixed finite time). Note that $q(x)=-\lambda|x|^{-\alpha}$ does not belong to the Kato class. As shown in Theorem 1.1, the heat kernel $\tilde{p}(t, x, y)$ associated with the Schrödinger operator $\mathcal{L}$ given by Eq. 1.1 exhibits behaviour which is different from that of the case that $q(x)=-\lambda|x|^{-\gamma}$ with $\gamma \in$ $(0, \alpha)$, which is in the Kato class. The study of heat kernel estimates for Schrödinger-type perturbations by the Hardy potential of fractional Laplacian is much more delicate.

In the classical case $\alpha=2$, the Schrödinger-type perturbations by the Hardy potential were considered for the first time by Baras and Goldstein [4]. They proved the existence of nontrivial nonnegative solutions of the classical heat equation $\partial_{t}=\Delta+\kappa|x|^{-2}$ in $\mathbb{R}^{d}$ for $0 \leq \kappa \leq(d-2)^{2} / 4$, and nonexistence of such solutions, that is explosion, for bigger constants $\kappa$. Sharp upper and lower bounds for the heat kernel of the Schrödinger operator $\Delta+\kappa|x|^{-2}$ were obtained by Liskevich and Sobol [29, p. 365 and Examples 3.8, 4.5 and 4.10] for $0<\kappa<(d-2)^{2} / 4$. Milman and Semenov proved the upper and lower bounds for $\kappa \leq(d-2)^{2} / 4$, see [30, Theorem 1] and [31]. In this paper, they also allowed $\kappa<0$ and obtained the sharp upper and lower bounds for the perturbed kernel (see [30, Theorem 2 and Corollary 4]). See [23] and the references therein for the recent works of this topic. 
For $\alpha \in(0, d \wedge 2)$ the Schrödinger operator $\mathcal{L}$ with $\kappa \geq 0$ attains recently more and more interest. In $[1,2]$ for $\kappa>\kappa^{*}:=\frac{2^{\alpha} \Gamma((d+\alpha) / 4)^{2}}{\Gamma((d-\alpha) / 4)^{2}}$ the phenomenon of instantaneous blow up of heat kernel was proven. In [5], the author gives the upper bound for the heat kernel of $\mathcal{L}$ with the Dirichlet conditions on bounded open subsets of $\mathbb{R}^{d}$. In the recent paper [8], the following sharp estimates for the heat kernel $\tilde{p}(t, x, y)$ of $\mathcal{L}$ were obtained. For $0 \leq \kappa \leq \kappa^{*}$, there is a unique constant $\delta \in[0,(d-\alpha) / 2]$ such that for all $t>0$ and $x, y \in \mathbb{R}_{0}^{d}:=\mathbb{R}^{d} \backslash\{0\}$,

$$
\tilde{p}(t, x, y) \approx\left(1+t^{\delta / \alpha}|x|^{-\delta}\right)\left(1+t^{\delta / \alpha}|y|^{-\delta}\right) p(t, x, y) .
$$

Note that since the singularity of the function $\mathbb{R}^{d} \ni x \mapsto \kappa|x|^{-\alpha}$ at the origin is critical, $\tilde{p}(t, x, y)$ is not comparable with the unperturbed kernel $p(t, x, y)$. Like in Theorem 1.1, the choice of $\kappa$ influences the growth rate or the decay rate of the heat kernel at the origin. This rate is represented by the function $|x|^{-\delta}$, where $\delta$ is connected with $\kappa$ via the formula $\kappa=\frac{2^{\alpha} \Gamma\left(\frac{\alpha+\delta}{2}\right) \Gamma\left(\frac{d-\delta}{2}\right)}{\Gamma\left(\frac{\delta}{2}\right) \Gamma\left(\frac{d-\delta-\alpha}{2}\right)}$ (compared with Eq. 1.2).

Remark 1.2 Theorem 1.1 and [8, Theorem 1.1] (the main result of [8]) can be stated together as follows. For any $-\infty<\kappa \leq \kappa^{*}=\frac{2^{\alpha} \Gamma((d+\alpha) / 4)^{2}}{\Gamma((d-\alpha) / 4)^{2}}$, the heat kernel $\tilde{p}(t, x, y)$ corresponding to the Schrödinger operator $\Delta^{\alpha / 2}+\kappa|x|^{-\alpha}$ satisfies

$$
\tilde{p}(t, x, y) \approx\left(1+\frac{t^{1 / \alpha}}{|x|^{\alpha}}\right)^{\delta}\left(1+\frac{t^{1 / \alpha}}{|y|^{\alpha}}\right)^{\delta} p(t, x, y), \quad t>0, x, y \in \mathbb{R}_{0}^{d},
$$

where $\delta \in\left(-\alpha, \frac{d-\alpha}{2}\right]$ is uniquely determined by

$$
\kappa=\frac{2^{\alpha} \Gamma\left(\frac{\alpha+\delta}{2}\right) \Gamma\left(\frac{d-\delta}{2}\right)}{\Gamma\left(\frac{\delta}{2}\right) \Gamma\left(\frac{d-\delta-\alpha}{2}\right)} .
$$

In this setting, Theorem 1.1 may be treated as both a fractional counterpart of the result obtained in [30] and the extension of Eq. 1.4 to negative values of $\kappa$. Here, we would like to point out one difference between the cases $\alpha=2$ and $\alpha<2$ for $\kappa<0$. The general form of the estimate in both cases is similar, i.e., the perturbed kernel $\tilde{p}(t, x, y)$ is comparable with the unperturbed kernel $p(t, x, y)$ multiplied by some weighted functions. However, in [30, Theorem 2 and Corollary 4], for $\alpha=2$, the exponent of the weighted function is equal to $\delta=\frac{\sqrt{(d-2)^{2}-4 \kappa}-(d-2)}{2}$ and converges to infinity as $\kappa \rightarrow-\infty$. In our case $\alpha<2$, as it was mentioned below the statement of Theorem 1.1, $\delta \rightarrow \alpha$ for $\kappa \rightarrow-\infty$. Since $q(x)=$ $\kappa|x|^{-\alpha}$ is negative and does not belong to any Kato class on $\mathbb{R}^{d}$, the construction and proofs of the estimates of $\tilde{p}(t, x, y)$ are very delicate. In particular, we cannot use the perturbation series (at least for large values of $-\kappa)$ to construct $\tilde{p}(t, x, y)$ as used in [7-9]. That is why we will consider the Dirichlet fractional Laplacian operator $\Delta^{\alpha / 2}$ on $\mathbb{R}_{0}^{d}=\mathbb{R}^{d} \backslash\{0\}$ and via the Feyman-Kac formula, we construct $\tilde{p}(t, x, y)$ on $(0, \infty) \times \mathbb{R}_{0}^{d} \times \mathbb{R}_{0}^{d}$. Hence, the operator $\mathcal{L}$ with negative values of $\kappa$ also enjoys some probabilistic meaning. Roughly speaking, it is connected with a symmetric $\alpha$-stable process with killing in terms of the negative potential $\kappa|x|^{-\alpha}$, which strongly affects the behaviour of $\tilde{p}(t, x, y)$ for $x$ and $y$ near 0 . It turns out that due to the strong singularity of $q(x)$ at 0 , the heat kernel (or the transition density function) $\tilde{p}(t, x, y)$ is equal to 0 when $x=0$ or $y=0$. In consequence, the kernel $\tilde{p}(t, x, y)$ defined on $(0, \infty) \times \mathbb{R}_{0}^{d} \times \mathbb{R}_{0}^{d}$ may be continuously extended to $(0, \infty) \times \mathbb{R}^{d} \times \mathbb{R}^{d}$. 
We note that Theorem 1.1 was proved independently in a very recent paper [17]. In the proofs, the authors use generally probabilistic tools. In our paper we propose a different method. Although the perturbed kernel $\tilde{p}(t, x, y)$ is defined by the Feyman-Kac formula, in the proofs we apply only analytical tools. For upper bounds, we generally use the ChapmanKolmogorov equation and the method of "self-improving estimates" (see the proofs of Proposition 3.1 and Theorem 3.5, see also the proof of [25, Theorem 1.1]). Roughly speaking, to show the inequality $f(x) \leq C F(x)$, we first show that $f(x) \leq g_{1}(x)+c_{1} F(x)$, where $g_{1}(x)$ is in some sense small. Next, by plugging this estimate to the proper functional inequality on $f$, we get the improved estimate of the form $f(x) \leq g_{n}(x)+c_{n} F(x)$, where $g_{n}(x) \rightarrow 0$ as $n \rightarrow \infty$ and $\sup _{n \in \mathbb{N}_{+}} c_{n}<\infty$. By passing with $n$ to infinity we obtain the desired estimate. To obtain lower bounds we use the generally well known estimate from Lemma 3.8 and upper bound estimates. Although the estimate from Lemma 3.8 is generally well known, we couldn't find the proper reference with the assumptions on the potential satisfied by $q(x)$. We note that the setting of [17] is more general than the present paper. From the other side, we give more details about the kernel $\tilde{p}(t, x, y)$, see e.g. Theorem 2.4. We also note that in our paper we show the straightforward dependence between the exponent $\delta$ and the potential $q(x)$, while in [17, Theorem 3.9] this dependence, given by double integral, is much more complicated.

The paper is organized as follows. In Section 2, we construct $\tilde{p}(t, x, y)$ and prove some basic properties of this kernel. In Section 3, we give the proof of Theorem 1.1. First, we prove upper bounds in Theorem 3.5. Next, we show lower bounds in Theorem 3.13 and joint continuity (Theorem 3.16). We end this section with short discussion on Dirichlet forms associated with the Schrödinger operator $\mathcal{L}$ given by Eq. 1.1. Finally, in the Appendix, we present the proof Lemma 3.8.

Throughout the paper, we write $f \approx g$ for $f, g \geq 0$, if there is a constant $c \geq 1$ such that $c^{-1} f \leq g \leq c f$ on their common domain. The constants $c, C, c_{i}$, whose exact values are unimportant, are changed in each statement and proof. Let $B(x, r)$ be the open ball with center $x \in \mathbb{R}^{d}$ and radius $r>0$. As usual we write $a \wedge b:=\min (a, b)$ and $a \vee b:=\max (a, b)$.

\section{Preliminary Estimates}

\subsection{Fractional Laplacian and Rotationally Symmetric $\alpha$-Stable Lévy Process}

Let

$$
v(z)=\frac{\alpha 2^{\alpha-1} \Gamma((d+\alpha) / 2)}{\pi^{d / 2} \Gamma(1-\alpha / 2)}|z|^{-d-\alpha}, \quad z \in \mathbb{R}^{d} .
$$

For (smooth and compactly supported) test function $\varphi \in C_{c}^{\infty}\left(\mathbb{R}^{d}\right)$, we define the fractional Laplacian by

$$
\Delta^{\alpha / 2} \varphi(x)=-(-\Delta)^{\alpha / 2} \varphi(x):=\lim _{\varepsilon \downarrow 0} \int_{\{|z|>\varepsilon\}}[\varphi(x+z)-\varphi(x)] v(z) d z, \quad x \in \mathbb{R}^{d} .
$$

In terms of the Fourier transform (see [20, Section 1.1.2]), $\widehat{\Delta^{\alpha / 2} \varphi}(\xi)=-|\xi|^{\alpha} \hat{\varphi}(\xi)$. Denote by $p(t, x, y)$ the heat kernel (or the fundamental function) of $\Delta^{\alpha / 2}$ (or equivalently, the transition density function of a (rotationally) symmetric $\alpha$-stable Lévy process $\left.\left(X_{t}\right)_{t \geq 0}\right)$. It 
is well known that $p(t, x, y)$ is symmetric in the sense that $p(t, x, y)=p(t, y, x)$ for any $t>0$ and $x, y \in \mathbb{R}^{d}$, and enjoys the following scaling property

$$
p(t, x, y)=t^{-d / \alpha} p\left(1, t^{-1 / \alpha} x, t^{-1 / \alpha} y\right), \quad t>0, x, y \in \mathbb{R}^{d} .
$$

Moreover,

$$
p(t, x, y) \approx t^{-d / \alpha} \wedge \frac{t}{|x-y|^{d+\alpha}}, \quad t>0, x, y \in \mathbb{R}^{d} .
$$

We also note that $p(t, x, y)$ is a function of $t$ and $x-y$, so sometimes we also write it as $p(t, x-y)$, i.e. $p(t, x, y)=p(t, x-y)$. See [6] for more details.

\subsection{Fractional Laplacian Schrödinger Operator and Feynman-Kac Formula}

In this part, we apply some results from [19, Chapter 2] to the operator $\mathcal{L}=\Delta^{\alpha / 2}+q$ given by Eq. 1.1, where $q(x)=\kappa_{\delta}|x|^{-\alpha}<0$. Let $\mathbb{R}_{0}^{d}:=\mathbb{R}^{d} \backslash\{0\}$. We first recall [19, Chapter 2, Definition 2.1]. A nonnegative Borel measurable function $V$ on $\mathbb{R}_{0}^{d}$ is said to belong to the Kato class $\mathcal{K}_{\alpha}$, if

$$
\lim _{t \rightarrow 0} \sup _{x \in \mathbb{R}_{0}^{d}} \int_{0}^{t} \int_{\mathbb{R}_{0}^{d}} p(s, x, y) V(y) d y d s=0 .
$$

A nonnegative Borel measurable function $V$ on $\mathbb{R}_{0}^{d}$ is said to belong to the local Kato class $\mathcal{K}_{\alpha, \text { loc }}$, if $V \mathbb{1}_{D} \in \mathcal{K}_{\alpha}$ for all compact subsets $D$ of $\mathbb{R}_{0}^{d}$. A Borel measurable function $V$ on $\mathbb{R}_{0}^{d}$ is said to belong to the Kato-Feller class, if its positive part $V_{+}:=\max \{V, 0\} \in \mathcal{K}_{\alpha}$ and its negative part $V_{-}:=\max \{-V, 0\} \in \mathcal{K}_{\alpha, \text { loc }}$. (Different from [19], in the present setting we start from the nonpositive definite operator $\Delta^{\alpha / 2}+q$, and so we make the corresponding changes in the definition of the Kato-Feller class.) It is easily seen from [8, Lemma 2.3] that

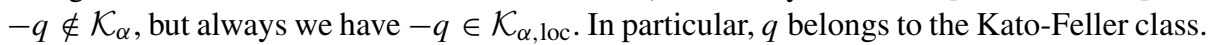

In the following, we will restrict ourselves on the killed subprocess of the symmetric $\alpha$-stable Lévy process $\left(X_{t}\right)_{t \geq 0}$ upon exiting $\mathbb{R}_{0}^{d}$ (or hitting the origin), i.e.,

$$
X_{t}^{\mathbb{R}_{0}^{d}}:= \begin{cases}X_{t}, & \text { if } t<\tau_{\mathbb{R}_{0}^{d}}, \\ 0, & \text { if } t \geq \tau_{\mathbb{R}_{0}^{d}}\end{cases}
$$

where $\tau_{\mathbb{R}_{0}^{d}}:=\inf \left\{t \geq 0: X_{t} \notin \mathbb{R}_{0}^{d}\right\}=\inf \left\{t \geq 0: X_{t}=0\right\}$. By the strong Markov property of the process $\left(X_{t}\right)_{t \geq 0}$, it is easy to see that the process $\left(X_{t}^{\mathbb{R}_{0}^{d}}\right)_{t \geq 0}$ has a transition density

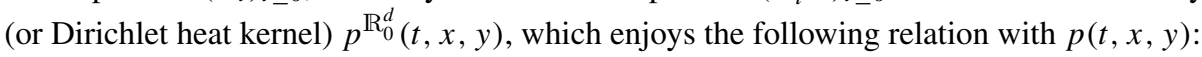

$$
p^{\mathbb{R}_{0}^{d}}(t, x, y)=p(t, x, y)-\mathbb{E}^{x}\left[p\left(t-\tau_{\mathbb{R}_{0}^{d}}, X_{\tau_{\mathbb{R}_{0}^{d}}}, y\right) \mathbb{1}_{\left\{t \geq \tau_{\mathbb{R}_{0}^{d}}\right\}}\right], \quad x, y \in \mathbb{R}_{0}^{d} .
$$

According to [26, Theorem 1. c), p. 5-6; Theorem 3, p. 8] and the assumption that $0<\alpha<$ $d, \mathbb{P}^{x}\left(\tau_{\mathbb{R}_{0}^{d}}<\infty\right)=0$ for all $x \in \mathbb{R}_{0}^{d}$. Consequently,

$$
p^{\mathbb{R}_{0}^{d}}(t, x, y)=p(t, x, y), \quad t>0, x, y \in \mathbb{R}_{0}^{d} .
$$

It is well known that for every $t>0$ the function $p(t, \cdot, \cdot)$ is continuous on $\mathbb{R}^{d} \times \mathbb{R}^{d}$, and $p(t, x, y)$ satisfies the following Chapman-Kolmogorov equation

$$
p(t+s, x, y)=\int_{\mathbb{R}^{d}} p(t, x, z) p(s, z, y) d z, \quad t, s>0, x, y \in \mathbb{R}^{d} .
$$

A continuous function $f$ on $\mathbb{R}_{0}^{d}$ is said to belong to $C_{\infty}\left(\mathbb{R}_{0}^{d}\right)$, if for every $\varepsilon>0$ there is a compact set $K \subset \mathbb{R}_{0}^{d}$ such that $|f(x)| \leq \varepsilon$ for all $x \notin K$. Regard $\mathcal{L}=\Delta^{\alpha / 2}+q$ as the 
operator defined on $C_{\infty}\left(\mathbb{R}_{0}^{d}\right)$; that is, we consider a negative perturbation of the fractional Laplacian on $\mathbb{R}_{0}^{d}$ (with the Dirichlet boundary condition at $\{0\}$ ). Therefore, according to [19, Theorem 2.5], the operator $\mathcal{L}=\Delta^{\alpha / 2}+q$ generates a strongly continuous and positivity preserving semigroup $\left(\tilde{P}_{t}\right)_{t \geq 0}$ on $C_{\infty}\left(\mathbb{R}_{0}^{d}\right)$, which is given by

$$
\tilde{P}_{t} f(x)=\int \tilde{p}(t, x, y) f(y) d y, \quad f \in C_{\infty}\left(\mathbb{R}_{0}^{d}\right), x \in \mathbb{R}_{0}^{d},
$$

where the kernel $\tilde{p}(t, x, y)$ satisfies the Chapman-Kolmogorov equation too, i.e.,

$$
\tilde{p}(t+s, x, y)=\int \tilde{p}(t, x, z) \tilde{p}(s, z, y) d z, \quad t, s>0, x, y \in \mathbb{R}_{0}^{d} .
$$

Additionally, for $t>0$, we put $\tilde{p}(t, x, y)=0$, whenever $x=0$ or $y=0$. Moreover, $\left(\tilde{P}_{t}\right)_{t \geq 0}$ also acts as a strongly continuous semigroup in $L^{p}\left(\mathbb{R}_{0}^{d} ; d x\right)$ for all $1 \leq p<\infty$, and, in $L^{2}\left(\mathbb{R}_{0}^{d} ; d x\right)$ the semigroup $\left(\tilde{P}_{t}\right)_{t \geq 0}$ is self-adjoint. Meanwhile, $\left(\tilde{P}_{t}\right)_{t \geq 0}$ is given via the Feynman-Kac formula:

$$
\begin{aligned}
\tilde{P}_{t} f(x) & =\mathbb{E}^{x}\left(f\left(X_{t}^{\mathbb{R}_{0}^{d}}\right) e^{\int_{0}^{t} q\left(X_{s}^{\mathbb{R}_{0}^{d}}\right) d s}\right) \\
& =\mathbb{E}^{x}\left(f\left(X_{t}\right) e^{\int_{0}^{t} q\left(X_{s}\right) d s}\right), \quad f \in C_{\infty}\left(\mathbb{R}_{0}^{d}\right), t>0, x \in \mathbb{R}_{0}^{d} .
\end{aligned}
$$

We used here that $\mathbb{P}^{x}\left(\tau_{\mathbb{R}_{0}^{d}}<\infty\right)=0$ for all $x \in \mathbb{R}_{0}^{d}$.

Due to $q(x)<0$ on $\mathbb{R}_{0}^{d}$ again, it follows from Eqs. 2.3 and 2.6 that

$$
\tilde{p}(t, x, y) \leq p(t, x, y), \quad t>0, x, y \in \mathbb{R}^{d} .
$$

Since $\left(\tilde{P}_{t}\right)_{t \geq 0}$ is self-adjoint in $L^{2}\left(\mathbb{R}_{0}^{d} ; d x\right)$,

$$
\tilde{p}(t, x, y)=\tilde{p}(t, y, x), \quad t>0, x, y \in \mathbb{R}^{d} .
$$

According to [19, Propositions 5.2 and 5.3] and their proofs, $\tilde{p}(t, x, y)$ will satisfy the following Duhamel's formula:

$$
\begin{aligned}
\tilde{p}(t, x, y) & =p(t, x, y)+\int_{0}^{t} \int_{\mathbb{R}^{d}} p(t-s, x, z) q(z) \tilde{p}(s, z, y) d z d s \\
& =p(t, x, y)+\int_{0}^{t} \int_{\mathbb{R}^{d}} \tilde{p}(t-s, x, z) q(z) p(s, z, y) d z d s
\end{aligned}
$$

for all $t>0$ and $x, y \in \mathbb{R}_{0}^{d}$.

Next, we show that $\tilde{p}(t, x, y)$ enjoys the same scaling property as $p(t, x, y)$.

Lemma 2.1 For any $t>0$ and $x, y \in \mathbb{R}^{d}$,

$$
\tilde{p}(t, x, y)=t^{-d / \alpha} \tilde{p}\left(1, x t^{-1 / \alpha}, y t^{-1 / \alpha}\right) .
$$

Proof We only consider the case that $x, y \in \mathbb{R}_{0}^{d}$; otherwise, the statement holds trivially. Recall that for the symmetric $\alpha$-stable process $\left(X_{t}\right)_{t \geq 0}$, the processes $\left(X_{u t}\right)_{t \geq 0}$ and $\left(t^{1 / \alpha} X_{u}\right)_{t \geq 0}$ enjoy the same law for any fixed $u>0$. For fixed $t>0$, set $\hat{X}_{u}=X_{u t}$ for $u \geq 0$. Then, by Eq. 2.6, for any $f \in C_{\infty}\left(\mathbb{R}_{0}^{d}\right), t>0$ and $x \in \mathbb{R}_{0}^{d}$,

$$
\begin{aligned}
\tilde{P}_{t} f(x) & =\mathbb{E}^{x}\left(f\left(X_{t}\right) e^{\int_{0}^{t} q\left(X_{s}\right) d s}\right)=\mathbb{E}^{x}\left(f\left(\hat{X}_{1}\right) e^{\int_{0}^{t} q\left(\hat{X}_{s / t}\right) d s}\right)=\mathbb{E}^{x}\left(f\left(\hat{X}_{1}\right) e^{t \int_{0}^{1} q\left(\hat{X}_{u}\right) d u}\right) \\
& =\mathbb{E}^{t^{-1 / \alpha} x}\left(f\left(t^{1 / \alpha} X_{1}\right) e^{t \int_{0}^{1} q\left(t^{1 / \alpha} X_{u}\right) d u}\right)=\mathbb{E}^{t^{-1 / \alpha} x}\left(f\left(t^{1 / \alpha} X_{1}\right) e^{\int_{0}^{1} q\left(X_{u}\right) d u}\right),
\end{aligned}
$$


where in the last equality we used the fact that $q(x)=\kappa_{\delta}|x|^{-\alpha}$. Hence, the desired assertion follows from the equality above.

\subsection{Integral Analysis for Fractional Laplacian Schrödinger Operator}

Lemma 2.2 Let $\beta \in(0,2)$. Then,

$$
|x|^{\beta}=2^{d+\beta} \pi^{d / 2} \frac{\Gamma\left(\frac{d+\beta}{2}\right)}{\Gamma\left(\frac{d+\beta}{\alpha}\right)\left|\Gamma\left(\frac{-\beta}{2}\right)\right|} \int_{0}^{\infty}[p(t, 0)-p(t, x)] t^{\frac{d-\alpha+\beta}{\alpha}} d t
$$

where $\Gamma(-\beta / 2)=\int_{0}^{\infty} t^{-1-\beta / 2}\left(e^{-t}-1\right) d t$.

Proof We follow the method used in the proof of [7, Proposition 5]. First, let $\eta_{t}(s)$ be the density function of the distribution of the $\alpha / 2$-stable subordinator at time $t$. Let $g_{t}(x)=$ $(4 \pi t)^{-d / 2} e^{-|x|^{2} /(4 t)}$. Then,

$$
p(t, x)=\int_{0}^{\infty} g_{s}(x) \eta_{t}(s) d s .
$$

By [7, (24)], for $\gamma<d / 2-1$,

$$
\int_{0}^{\infty} g_{s}(x) s^{\gamma} d s=4^{-\gamma-1} \pi^{-d / 2} \Gamma(d / 2-\gamma-1)|x|^{2 \gamma-d+2} .
$$

Then, by integrating by parts, for $d / 2-1<\gamma<d / 2$, we get

$$
\begin{aligned}
\int_{0}^{\infty}\left(g_{s}(0)-g_{s}(x)\right) s^{\gamma} d s & =(4 \pi)^{-d / 2} \int_{0}^{\infty}\left(1-e^{-\frac{|x|^{2}}{4 s}}\right) s^{\gamma-d / 2} d s \\
& =\frac{(4 \pi)^{-d / 2}}{\gamma+1-d / 2} \int_{0}^{\infty} \frac{|x|^{2}}{4 s^{2}} e^{-\frac{|x|^{2}}{4 s}} s^{\gamma+1-d / 2} d s \\
& =\frac{|x|^{2}}{4(\gamma+1-d / 2)} \int_{0}^{\infty} g_{s}(x) s^{\gamma-1} d s \\
& =4^{-\gamma-1} \pi^{-d / 2} \frac{\Gamma(d / 2-\gamma)}{\gamma+1-d / 2}|x|^{2 \gamma-d+2} .
\end{aligned}
$$

Note that, for any $\gamma>-1$,

$$
\int_{0}^{\infty} t^{\gamma} \eta_{t}(s) d t=\frac{\Gamma(\gamma+1)}{\Gamma\left(\frac{\alpha(\gamma+1)}{2}\right)} s^{\frac{\alpha(\gamma+1)}{2}-1},
$$

see [7, (23)]. (Note that the condition that $\gamma<d / \alpha-1$ is not required in the proof of [7, (23)].) We further obtain

$$
\begin{aligned}
\int_{0}^{\infty}[p(t, 0)-p(t, x)] t^{\frac{d-\alpha+\beta}{\alpha}} d t & =\int_{0}^{\infty} \int_{0}^{\infty}\left[g_{s}(0)-g_{s}(x)\right] \eta_{t}(s) t^{\frac{d-\alpha+\beta}{\alpha}} d t d s \\
& =\frac{\Gamma\left(\frac{d+\beta}{\alpha}\right)}{\Gamma\left(\frac{d+\beta}{2}\right)} \int_{0}^{\infty}\left[g_{s}(0)-g_{s}(x)\right] s^{\frac{d+\beta-2}{2}} d s \\
& =2^{-d-\beta} \pi^{-d / 2} \frac{\Gamma\left(\frac{d+\beta}{\alpha}\right)}{\Gamma\left(\frac{d+\beta}{2}\right)} \frac{\Gamma\left(\frac{2-\beta}{2}\right)}{\frac{\beta}{2}}|x|^{\beta} .
\end{aligned}
$$


Since $\Gamma\left(\frac{2-\beta}{2}\right)=-\frac{\beta}{2} \Gamma\left(-\frac{\beta}{2}\right)$, we get the assertion of the lemma.

We recall from $[7,(25)]$ that for any $\beta \in(0, d)$,

$$
|x|^{-\beta}=2^{d-\beta} \pi^{d / 2} \frac{\Gamma\left(\frac{d-\beta}{2}\right)}{\Gamma\left(\frac{d-\beta}{\alpha}\right) \Gamma\left(\frac{\beta}{2}\right)} \int_{0}^{\infty} p(t, x) t^{\frac{d-\alpha-\beta}{\alpha}} d t, \quad x \in \mathbb{R}^{d} .
$$

Thus, Eq. 2.8 may be treated as an extension of the formula (2.9) to negative $\beta$. Note that in the proof of Eq. 2.8 we have to use a compensated kernel $p(t, 0)$ to ensure convergence of the integral involved.

Now, let $\beta \in(0, \alpha)$. By Eq. 2.9,

$$
|x|^{\beta-\alpha}=2^{d+\beta-\alpha} \pi^{d / 2} \frac{\Gamma\left(\frac{d+\beta-\alpha}{2}\right)}{\Gamma\left(\frac{d+\beta-\alpha}{\alpha}\right) \Gamma\left(\frac{\alpha-\beta}{2}\right)} \int_{0}^{\infty} p(t, x) t^{\frac{d-2 \alpha+\beta}{\alpha}} d t .
$$

On the other hand, let $f(r)=c r^{(d-\alpha+\beta) / \alpha}$ with

$$
c=2^{d+\beta} \pi^{d / 2} \frac{\Gamma\left(\frac{d+\beta}{2}\right)}{\Gamma\left(\frac{d+\beta}{\alpha}\right)\left|\Gamma\left(\frac{-\beta}{2}\right)\right|} .
$$

Then, according to Eq. 2.8,

$$
|x|^{\beta}=\int_{0}^{\infty}[p(t, 0)-p(t, x)] f(t) d t .
$$

Combining two equations above together, we will find that

$$
-\kappa_{\beta}|x|^{-\alpha}=\frac{\int_{0}^{\infty} p(r, x) f^{\prime}(r) d r}{\int_{0}^{\infty}[p(r, 0)-p(r, x)] f(r) d r},
$$

where

$$
\kappa_{\beta}=\frac{2^{\alpha} \Gamma\left(\frac{\alpha-\beta}{2}\right) \Gamma\left(\frac{d+\beta}{2}\right)}{\Gamma\left(\frac{-\beta}{2}\right) \Gamma\left(\frac{d+\beta-\alpha}{2}\right)} .
$$

In particular,

$$
-\kappa_{\beta}|x|^{\beta-\alpha}=\int_{0}^{\infty} p(r, x) f^{\prime}(r) d r .
$$

We note that $\kappa_{\beta}<0$ for any $\beta \in(0, \alpha)$ and $\lim _{\beta \rightarrow \alpha} \kappa_{\beta}=-\infty$. For convenience, let $\kappa_{0}=0$. Moreover, write

$$
\kappa_{\beta}=-\frac{2^{\alpha} \Gamma\left(\frac{\alpha-\beta}{2}\right) \Gamma\left(\frac{d+\beta}{2}\right) \frac{\beta}{2}}{\Gamma\left(\frac{2-\beta}{2}\right) \Gamma\left(\frac{d+\beta-\alpha}{2}\right)},
$$

and let

$$
r(t)=\frac{\Gamma\left(\frac{\alpha}{2}-t\right) \Gamma\left(\frac{d}{2}+t\right)}{\Gamma\left(\frac{d-\alpha}{2}+t\right) \Gamma(1-t)}, \quad 0<t<\alpha / 2 .
$$

Then, using the formula

$$
\frac{\Gamma^{\prime}(x)}{\Gamma(x)}=-\gamma-\sum_{k=0}^{\infty}\left(\frac{1}{x+k}-\frac{1}{1+k}\right), \quad x>0
$$


with the Euler-Mascheroni constant $\gamma$ (see [3, (1.2.13)]) and following the argument in the end of the proof for [7, Proposition 5], one can check that $r(t)$ is strictly increasing on $(0, \alpha / 2)$, and so $\beta \mapsto \kappa_{\beta}$ is strictly decreasing on $(0, \alpha)$.

Lemma 2.3 For $\beta \in(0, \alpha), t>0$ and $x \in \mathbb{R}^{d}$, we have

$$
\int_{\mathbb{R}^{d}} p(t, x, y)|y|^{\beta} d y=|x|^{\beta}-\kappa \beta \int_{0}^{t} \int_{\mathbb{R}^{d}} p(s, x, y)|y|^{\beta-\alpha} d y d s .
$$

Proof Let $f(r)=c r^{(d-\alpha+\beta) / \alpha}$ with the constant $c$ given by Eq. 2.10. By Eqs. 2.12 and 2.11 , for any $t>0$ and $x \in \mathbb{R}^{d}$,

$$
\begin{aligned}
-\kappa_{\beta} \int_{0}^{t} \int_{\mathbb{R}^{d}} p(s, x, y)|y|^{\beta-\alpha} d y d s & =\int_{0}^{t} \int_{0}^{\infty} p(s+r, x) f^{\prime}(r) d r d s \\
& =-\int_{0}^{t} \int_{0}^{\infty} \frac{\partial}{\partial s} p(s+r, x) f(r) d r d s \\
& =\int_{0}^{\infty}[p(r, x)-p(t+r, x)] f(r) d r \\
& =\int_{0}^{\infty}[p(r, x)-p(r, 0)+p(r, 0)-p(t+r, x)] f(r) d r \\
& =-|x|^{\beta}+\int_{0}^{\infty} \int_{\mathbb{R}^{d}} p(t, x, y)[p(r, 0)-p(r, y)] f(r) d y d r \\
& =-|x|^{\beta}+\int_{\mathbb{R}^{d}} p(t, x, y)|y|^{\beta} d y,
\end{aligned}
$$

where in the second equality we used the fact that

$$
\lim _{r \rightarrow \infty} p(s+r, x) f(r) \leq c_{1} \lim _{r \rightarrow \infty}(s+r)^{-d / \alpha} f(r)=0 .
$$

This completes the proof.

Set $h_{\beta}(x)=|x|^{\beta}$. Letting $t \rightarrow 0$ in Eq. 2.13, informally it holds that

$$
\left(\Delta^{\alpha / 2}+\kappa_{\beta}|x|^{-\alpha}\right) h_{\beta}(x)=0
$$

for all $x \in \mathbb{R}^{d}$. That is, the function $h_{\beta}$ is harmonic with respect to the operator $\Delta^{\alpha / 2}+$ $\kappa_{\beta}|x|^{-\alpha}$.

From now, we will fix $\delta \in(0, \alpha)$, and write $\kappa_{\delta}$ as $\kappa$ for simplicity. The following theorem is an analog of [8, Theorem 3.1]. Since there is no problem with convergence of the integrals involved, the proof is much simpler than that of [8, Theorem 3.1].

Theorem 2.4 For $\beta \in(0, \alpha), t>0$ and $x \in \mathbb{R}^{d}$, we have

$$
\int_{\mathbb{R}^{d}} \tilde{p}(t, x, y)|y|^{\beta} d y=|x|^{\beta}+\left(\kappa-\kappa_{\beta}\right) \int_{0}^{t} \int_{\mathbb{R}^{d}} \tilde{p}(s, x, y)|y|^{\beta-\alpha} d y d s .
$$

In particular, for any $t>0$ and $x \in \mathbb{R}^{d}$,

$$
\int_{\mathbb{R}^{d}} \tilde{p}(t, x, y)|y|^{\delta} d y=|x|^{\delta} .
$$


The assertion (2.16) states that $h_{\delta}(x)=|x|^{\delta}$ is an invariant function for the semigroup $\left(\tilde{P}_{t}\right)_{t \geq 0}$ and so it is harmonic, which justifies (2.14) rigorously. On the other hand, note that Eq. 2.14 implies that

$$
\left[\Delta^{\alpha / 2}+\kappa|x|^{-\alpha}\right] h_{\beta}(x)=\left(\kappa-\kappa_{\beta}\right)|x|^{\beta-\alpha}, \quad x \in \mathbb{R}^{d} .
$$

From this one can deduce (2.15) informally (but directly) via the Feynman-Kac semigroup associated with $\Delta^{\alpha / 2}+\kappa|x|^{-\alpha}$.

Proof of Theorem 2.4 When $x=0$, both sides of Eqs. 2.15 and 2.16 are equal to zero, since $\tilde{p}(t, 0, y)=0$ for all $t>0$ and $y \in \mathbb{R}^{d}$. Below, we consider the case that $x \in \mathbb{R}_{0}^{d}$. By Eqs. 2.7 and 2.13, for any $t>0$ and $x \in \mathbb{R}_{0}^{d}$,

$$
\begin{aligned}
& -\kappa_{\beta} \int_{0}^{t} \int_{\mathbb{R}^{d}} \tilde{p}(s, x, y)|y|^{\beta-\alpha} d y d s \\
= & -\kappa_{\beta} \int_{0}^{t} \int_{\mathbb{R}^{d}} p(s, x, y)|y|^{\beta-\alpha} d y d s \\
& -\kappa_{\beta} \int_{0}^{t} \int_{\mathbb{R}^{d}} \int_{u}^{t} \int_{\mathbb{R}^{d}} \tilde{p}(u, x, z) q(z) p(s-u, z, y)|y|^{\beta-\alpha} d z d u d y d s \\
= & -|x|^{\beta}+\int_{\mathbb{R}^{d}} p(t, x, y)|y|^{\beta} d y \\
& -\int_{0}^{t} \int_{\mathbb{R}^{d}} \tilde{p}(u, x, z) q(z)\left(|z|^{\beta}-\int_{\mathbb{R}^{d}} p(t-u, z, y)|y|^{\beta} d y\right) d z d u .
\end{aligned}
$$

Hence, according to Eq. 2.7 again, for any $t>0$ and $x \in \mathbb{R}_{0}^{d}$,

$$
\begin{aligned}
-\left(\kappa_{\beta}-\kappa\right) \int_{0}^{t} \int_{\mathbb{R}^{d}} \tilde{p}(s, x, y)|y|^{\beta-\alpha} d y d s= & -|x|^{\beta}+\int_{\mathbb{R}^{d}} p(t, x, y)|y|^{\beta} d y \\
& +\int_{\mathbb{R}^{d}}(\tilde{p}(t, x, y)-p(t, x, y))|y|^{\beta} d y \\
= & -|x|^{\beta}+\int_{\mathbb{R}^{d}} \tilde{p}(t, x, y)|y|^{\beta} d y,
\end{aligned}
$$

which proves (2.15). Now, Eq. 2.16 follows by taking $\beta=\delta$. The proof is complete.

Although the following lemma is not used in the proofs, we state it as one of the results. From this lemma we see that the right-hand side of Eq. 2.17 behaves near 0 as $-\log |x|$.

Lemma 2.5 For any $t>0$ and $x \in \mathbb{R}^{d}$, it holds that

$$
C \int_{\mathbb{R}^{d}} \tilde{p}(t, x, y)|y|^{\delta}(\log |y|-\log |x|) d y=\int_{0}^{t} \int_{\mathbb{R}^{d}} \tilde{p}(s, x, y)|y|^{\delta-\alpha} d y d s,
$$

where

$$
C:=\lim _{\beta \rightarrow \delta} \frac{\delta-\beta}{\kappa_{\beta}-\kappa}>0 .
$$


Proof By Eq. 2.15, Eq. 2.16 and the dominated convergence theorem,

$$
\begin{aligned}
\int_{0}^{t} \int_{\mathbb{R}^{d}} \tilde{p}(s, x, y)|y|^{\delta-\alpha} d y d s & =\lim _{\beta \rightarrow \delta} \frac{1}{\kappa_{\beta}-\kappa} \int_{\mathbb{R}^{d}} \tilde{p}(t, x, y)|y|^{\delta}\left(|x|^{\beta-\delta}-|y|^{\beta-\delta}\right) d y \\
& =C \int_{\mathbb{R}^{d}} \tilde{p}(t, x, y)|y|^{\delta}(\log |y|-\log |x|) d y
\end{aligned}
$$

proving the desired assertion.

\section{Two-Sided Estimates and Joint Continuity of $\tilde{p}(t, x, y)$}

\subsection{Upper Bounds of $\tilde{p}(1, x, y)$}

For any $t>0$ and $x \in \mathbb{R}^{d}$, define

$$
H(t, x)=\int_{\mathbb{R}^{d}} \tilde{p}(t, x, y) d y .
$$

Note that, by Lemma 2.1 , for all $t>0$ and $x \in \mathbb{R}^{d}$, we have

$$
H(t, x)=\int_{\mathbb{R}^{d}} t^{-d / \alpha} \tilde{p}\left(1, t^{-1 / \alpha} x, t^{-1 / \alpha} y\right) d y=H\left(1, t^{-1 / \alpha} x\right) .
$$

On the other hand, by the fact $0 \leq \tilde{p}(t, x, y) \leq p(t, x, y)$ for any $t>0$ and $x, y \in \mathbb{R}^{d}$, it also holds that

$$
0 \leq H(t, x) \leq \int_{\mathbb{R}^{d}} p(t, x, y) d y=1, \quad t>0, x \in \mathbb{R}^{d} .
$$

Proposition 3.1 There is a constant $C>0$ such that for all $x \in \mathbb{R}^{d}$,

$$
H(1, x) \leq C\left(1 \wedge|x|^{\delta}\right) .
$$

Proof By the Chapman-Kolmogorov Eq. 2.5 (which holds true for all $x, y \in \mathbb{R}^{d}$ ) and Eq. 3.1, for any $x, y \in \mathbb{R}^{d}$,

$$
\begin{aligned}
\tilde{p}(1, x, y) & =\int_{\mathbb{R}^{d}} \int_{\mathbb{R}^{d}} \tilde{p}(1 / 3, x, z) \tilde{p}(1 / 3, z, w) \tilde{p}(1 / 3, w, y) d w d z \\
& \leq \int_{\mathbb{R}^{d}} \int_{\mathbb{R}^{d}} \tilde{p}(1 / 3, x, z) \cdot c \cdot \tilde{p}(1 / 3, w, y) d w d z \\
& =c H(1 / 3, x) H(1 / 3, y)=c H\left(1,3^{1 / \alpha} x\right) H\left(1,3^{1 / \alpha} y\right),
\end{aligned}
$$

where the constant $c$ comes from the estimate $\tilde{p}(1 / 3, x, y) \leq p(1 / 3, x, y) \leq c$.

Denote by $|B(0, r)|$ the Lebesgue measure of $B(0, r)$. Fix $r>0$ small enough such that $\eta:=c|B(0, r)|<3^{-\delta / \alpha}$. According to Eqs. 2.16, 3.3 and 3.2, for any $x \in \mathbb{R}^{d}$, we have

$$
\begin{aligned}
H(1, x) & \leq \int_{B(0, r)} \tilde{p}(1, x, y) d y+\frac{1}{r^{\delta}} \int_{B(0, r)^{c}} \tilde{p}(1, x, y)|y|^{\delta} d y \\
& \leq \int_{B(0, r)} \tilde{p}(1, x, y) d y+M|x|^{\delta} \leq \int_{B(0, r)} c H\left(1,3^{1 / \alpha} x\right) d y+M|x|^{\delta}, \\
& =\eta H\left(1,3^{1 / \alpha} x\right)+M|x|^{\delta},
\end{aligned}
$$


where $M=r^{-\delta}$. Now, we can iterate the inequality (3.4) to obtain that for all $x \in \mathbb{R}^{d}$,

$$
\begin{aligned}
H(1, x) & \leq \eta H\left(1,3^{1 / \alpha} x\right)+M|x|^{\delta} \\
& \leq \eta\left[\eta H\left(1,3^{2 / \alpha} x\right)+M\left|3^{1 / \alpha} x\right|^{\delta}\right]+M|x|^{\delta} \\
& \leq \eta^{2}\left[\eta H\left(1,3^{3 / \alpha} x\right)+M\left|3^{2 / \alpha} x\right|^{\delta}\right]+M\left(1+\eta 3^{\delta / \alpha}\right)|x|^{\delta} \\
& \leq \cdots \\
& \leq \eta^{n} H\left(1,3^{n / \alpha} x\right)+M\left[1+\eta 3^{\delta / \alpha}+\cdots+\left(\eta 3^{\delta / \alpha}\right)^{n-1}\right]|x|^{\delta} .
\end{aligned}
$$

By Eq. 3.2, taking $n \rightarrow \infty$ in the inequality above, we get that for any $x \in \mathbb{R}^{d}$,

$$
H(1, x) \leq \frac{M}{1-\eta 3^{\delta / \alpha}}|x|^{\delta},
$$

yielding the desired assertion.

Applying Proposition 3.1 to Eq.3.3, we immediately get

Corollary 3.2 There is a constant $C>0$ such that

$$
\tilde{p}(1, x, y) \leq C\left(1 \wedge|x|^{\delta}\right)\left(1 \wedge|y|^{\delta}\right), \quad x, y \in \mathbb{R}^{d} .
$$

Next, we further refine upper bounds for $\tilde{p}(t, x, y)$.

Lemma 3.3 For any $t>0$ and $x, y \in \mathbb{R}^{d}$, we have

$$
\int_{B(y,|x-y| / 2)} p(t, x, z) p(t, z, y) d z \leq \frac{p(2 t, x, y)}{2} .
$$

Proof Fix $t>0$ and $x, y \in \mathbb{R}^{d}$. By symmetry,

$$
\int_{B(y,|x-y| / 2)} p(t, x, z) p(t, z, y) d z=\int_{B(x,|x-y| / 2)} p(t, x, z) p(t, z, y) d z .
$$

Hence, by Eq. 2.4,

$$
\begin{aligned}
& 2 \int_{B(y,|x-y| / 2)} p(t, x, z) p(t, z, y) d z \\
= & \int_{B(y,|x-y| / 2)} p(t, x, z) p(t, z, y) d z+\int_{B(x,|x-y| / 2)} p(t, x, z) p(t, z, y) d z \\
\leq & \int_{\mathbb{R}^{d}} p(t, x, z) p(t, z, y) d z=p(2 t, x, y) .
\end{aligned}
$$

This completes the proof.

Lemma 3.4 There exists a constant $M>0$ such that for any $t>0$ and $x, y \in \mathbb{R}^{d}$, we have

$$
\tilde{p}(t, x, y) \leq \int_{B(y,|x-y| / 2)} \tilde{p}(t / 2, x, z) \tilde{p}(t / 2, z, y) d z+\operatorname{Mh}(t, x) p(t, x, y),
$$

where $h(t, x)=t^{-\delta / \alpha}|x|^{\delta}$. 
Proof By Eq. 2.5, for any $t>0$ and $x, y \in \mathbb{R}^{d}$, we have

$$
\begin{aligned}
\tilde{p}(t, x, y)= & \int_{B(y,|x-y| / 2)} \tilde{p}(t / 2, x, z) \tilde{p}(t / 2, z, y) d z \\
& +\int_{B(y,|x-y| / 2)^{c}} \tilde{p}(t / 2, x, z) \tilde{p}(t / 2, z, y) d z .
\end{aligned}
$$

Note that, according to Eq. 3.1 and Proposition 3.1,

$$
\int_{\mathbb{R}^{d}} \tilde{p}(t, x, y) d y=H(t, x)=H\left(1, t^{-1 / \alpha} x\right) \leq \operatorname{ch}(t, x), \quad t>0, x \in \mathbb{R}^{d} .
$$

For $t>0$ and $x, y, z \in \mathbb{R}^{d}$ with $z \in B(y,|x-y| / 2)^{c}$, we have

$$
\tilde{p}(t / 2, z, y) \leq p(t / 2, z, y) \leq c_{1} p(t, x, y) .
$$

Hence, for any $t>0$ and $x, y \in \mathbb{R}^{d}$,

$$
\begin{aligned}
\int_{B(y,|x-y| / 2)^{c}} \tilde{p}(t / 2, x, z) \tilde{p}(t / 2, z, y) d z & \leq c_{1} p(t, x, y) \int_{B(y,|x-y| / 2)^{c}} \tilde{p}(t / 2, x, z) d z \\
& \leq c c_{1} h(t / 2, x) p(t, x, y) \\
& \leq M h(t, x) p(t, x, y),
\end{aligned}
$$

thus we get the assertion of the lemma.

Theorem 3.5 (Upper bounds) There is a constant $C>0$ such that for all $x, y \in \mathbb{R}^{d}$,

$$
\tilde{p}(1, x, y) \leq C\left(1 \wedge|x|^{\delta}\right)\left(1 \wedge|y|^{\delta}\right) p(1, x, y) .
$$

Proof Let $\eta=1 / 2$ and $v=2^{(\delta-\alpha) / \alpha}<1$. As in Lemma 3.4, denote $h(t, x)=t^{-\delta / \alpha}|x|^{\delta}$. Note that

$$
\eta h(t / 2, x)=\frac{1}{2}\left|(t / 2)^{-1 / \alpha} x\right|^{\delta}=2^{(\delta-\alpha) / \alpha}\left|t^{-1 / \alpha} x\right|^{\delta}=v h(t, x), \quad t>0, x \in \mathbb{R}^{d} .
$$

Let $M$ be the constant from Lemma 3.4. We will claim that for $n \geq 0$,

$$
\tilde{p}(t, x, y) \leq\left[\eta^{n+1}+\left(1+v+\ldots+v^{n}\right) M h(t, x)\right] p(t, x, y), \quad t>0, x, y \in \mathbb{R}^{d} .
$$

Indeed, for $t \in(0,1]$ and $x, y \in \mathbb{R}^{d}$, by Lemmas 3.3 and 3.4,

$$
\tilde{p}(t, x, y) \leq[\eta+M h(t, x)] p(t, x, y),
$$

where we used the fact $\tilde{p}(t, x, y) \leq p(t, x, y)$ for any $t>0$ and $x, y \in \mathbb{R}^{d}$. Next, we use induction. Suppose that

$$
\tilde{p}(t, x, y) \leq\left[\eta^{n}+\left(1+v+\ldots+v^{n-1}\right) M h(t, x)\right] p(t, x, y), \quad t>0, x, y \in \mathbb{R}^{d} .
$$

Then, for any $t>0$ and $x, y \in \mathbb{R}^{d}$, by Lemmas 3.4, 3.3 and Eq. 3.6,

$$
\begin{aligned}
\tilde{p}(t, x, y) \leq & \int_{B(y,|x-y| / 2)} \tilde{p}(t / 2, x, z) p(t / 2, z, y) d z+\operatorname{Mh}(t, x) p(t, x, y) \\
\leq & \int_{B(y,|x-y| / 2)}\left[\eta^{n}+\left(1+v+\ldots+v^{n-1}\right) \operatorname{Mh}(t / 2, x)\right] p(t / 2, x, z) p(t / 2, z, y) d z \\
& +M h(t, x) p(t, x, y) \\
\leq & {\left[\eta^{n}+\left(1+v+\ldots+v^{n-1}\right) M h(t / 2, x)\right] \eta p(t, x, y)+\operatorname{Mh}(t, x) p(t, x, y) } \\
\leq & {\left[\eta^{n+1}+\left(v+\ldots+v^{n}\right) M h(t, x)\right] p(t, x, y)+M h(t, x) p(t, x, y) } \\
= & {\left[\eta^{n+1}+\left(1+v+\ldots+v^{n}\right) \operatorname{Mh}(t, x)\right] p(t, x, y), }
\end{aligned}
$$


and Eq. 3.7 follows. Since $h(1, x)=|x|^{\delta}$, by letting $n$ to infinity in Eq. 3.7, we get

$$
\tilde{p}(1, x, y) \leq \frac{M}{1-v}|x|^{\delta} p(1, x, y), \quad x, y \in \mathbb{R}^{d} .
$$

In the following, we pass to the proof of Eq. 3.5. By symmetry, we may and do assume that $|x| \leq|y|$. For $x, y \in \mathbb{R}^{d}$ with $|y| \geq|x| \geq 1$, Eq. 3.5 follows by the estimate $\tilde{p}(1, x, y) \leq p(1, x, y)$. For $|x| \leq|y| \leq 1$, we use Corollary 3.2 and the estimate that $p(1, x, y) \geq c$. Finally, for $|x|<1 \leq|y|$, Eq. 3.5 follows by Eq. 3.8.

\subsection{Lower Bounds of $\tilde{p}(1, x, y)$}

We first begin with the following lemma, which is a consequence of Theorem 3.5.

Lemma 3.6 There is a constant $C>0$ such that

$$
H(1, x) \geq C\left(1 \wedge|x|^{\delta}\right), \quad x \in \mathbb{R}^{d} .
$$

Proof Let $R>0$ and $x \in B(0, R / 2)$. Then

$$
\int_{B(0, R)^{c}}|y|^{\delta} p(1, x, y) d y \leq c_{1} \int_{B(0, R)^{c}} \frac{|y|^{\delta}}{|y|^{d+\alpha}} d y=c_{2} R^{\delta-\alpha} \rightarrow 0 \quad \text { as } R \rightarrow \infty .
$$

Choose $R \geq 1$ be such that $c_{2} C R^{\delta-\alpha} \leq 1 / 2$, where $C$ is the constant given in Eq. 3.5. Then, by Eq. 2.16, for $r \geq R \geq 1$ and $x \in B(0, r / 2)$, we have

$$
\begin{aligned}
\int_{\mathbb{R}^{d}} \tilde{p}(1, x, y) d y & \geq r^{-\delta} \int_{B(0, r)} \tilde{p}(1, x, y)|y|^{\delta} d y \\
& =r^{-\delta}\left(|x|^{\delta}-\int_{B(0, r)^{c}} \tilde{p}(1, x, y)|y|^{\delta} d y\right) \\
& \geq r^{-\delta}\left(|x|^{\delta}-C \int_{B(0, r)^{c}}|x|^{\delta} p(1, x, y)|y|^{\delta} d y\right) \geq \frac{|x|^{\delta}}{2 r^{\delta}} .
\end{aligned}
$$

Hence, for $x \in B(0, R / 2)$, by Eq. 3.9,

$$
\int_{\mathbb{R}^{d}} \tilde{p}(1, x, y) d y \geq \frac{|x|^{\delta}}{2 R^{\delta}} \geq \frac{|x|^{\delta} \wedge 1}{2 R^{\delta}} ;
$$

for $x \in B(0, R / 2)^{c}$, taking $r=2|x|+1$ in Eq. 3.9, we can get that

$$
\int_{\mathbb{R}^{d}} \tilde{p}(1, x, y) d y \geq \frac{|x|^{\delta}}{2(2|x|+1)^{\delta}} \geq \frac{|x|^{\delta}}{2(4|x|)^{\delta}} \geq \frac{1}{4^{\delta+1}} .
$$

Combining both estimates above, we can prove the desired assertion.

To obtain lower bounds of $\tilde{p}(t, x, y)$, we need to consider the difference between $p(t, x, y)$ and $\tilde{p}(t, x, y)$. Motivated by Duhamel's formula (2.7), we define

$$
p_{1}(t, x, y)=\int_{0}^{t} \int_{\mathbb{R}^{d}} p(t-s, x, z)|z|^{-\alpha} p(s, z, y) d z d s, \quad t>0, x, y \in \mathbb{R}_{0}^{d} .
$$

It is easy to see that $p_{1}(t, x, y)$ also enjoys the same scaling property as $p(t, x, y)$, i.e.,

$$
p_{1}(t, x, y)=t^{-d / \alpha} p_{1}\left(1, x t^{-1 / \alpha}, y t^{-1 / \alpha}\right), \quad t>0, x, y \in \mathbb{R}_{0}^{d} .
$$


Let

$$
G(t, x)=\int_{0}^{t} \int_{\mathbb{R}^{d}} p(s, x, z)|z|^{-\alpha} d z d s, \quad t>0, x \in \mathbb{R}_{0}^{d} .
$$

By [8, Lemma 2.3],

$$
G(t, x) \approx \log \left(1+t|x|^{-\alpha}\right), \quad t>0, x \in \mathbb{R}_{0}^{d} .
$$

Lemma 3.7 For all $x, y \in \mathbb{R}_{0}^{d}$, we have

$$
p_{1}(1, x, y) \approx[G(1, x)+G(1, y)] p(1, x, y) .
$$

Proof By the 3P inequality (see [10, (9)] or [16, (2.11)]), for any $x, y \in \mathbb{R}_{0}^{d}$,

$$
\begin{aligned}
& \int_{0}^{1} \int_{\mathbb{R}^{d}} p(1-s, x, z)|z|^{-\alpha} p(s, z, y) d z d s \\
\leq & c_{1} p(1, x, y) \int_{0}^{1} \int_{\mathbb{R}^{d}}(p(1-s, x, z)+p(s, z, y))|z|^{-\alpha} d z d s \\
= & c_{1}(G(1, x)+G(1, y)) p(1, x, y),
\end{aligned}
$$

thus we get the upper bound.

Now, we pass to the lower bound. Since the function $|x| \mapsto \log \left(1+|x|^{-\alpha}\right)$ is decreasing in $|x|$, by Eq. 3.11 and the symmetry of $p(t, x, y)$ and $p_{1}(t, x, y)$, it suffices to prove

$$
p_{1}(1, x, y) \geq c_{2} G(1, x) p(1, x, y), \quad 0<|x| \leq|y| .
$$

First, let $|x|<2$. Then, by Eq. 2.2,

$$
\begin{aligned}
\int_{0}^{1 / 2} \int_{B(0,3)} p(s, x, z) \frac{1}{|z|^{\alpha}} d z d s & \geq \int_{0}^{1 / 2} \int_{B\left(x, s^{1 / \alpha}\right)} p(s, x, z) \frac{1}{|z|^{\alpha}} d z d s \\
& \geq c_{0} \geq c_{0} \int_{0}^{1 / 2} \int_{B(0,3)^{c}} p(s, x, z) \frac{1}{|z|^{\alpha}} d z d s
\end{aligned}
$$

and so

$$
\int_{0}^{1 / 2} \int_{\mathbb{R}^{d}} p(s, x, z) \frac{1}{|z|^{\alpha}} d z d s \approx \int_{0}^{1 / 2} \int_{B(0,3)} p(s, x, z) \frac{1}{|z|^{\alpha}} d z d s .
$$

Hence, for any $|x|<2$ and $|y|>4$,

$$
\begin{aligned}
p_{1}(1, x, y) & \geq \int_{0}^{1 / 2} \int_{B(0,3)} p(s, x, z) \frac{1}{|z|^{\alpha}} p(1-s, z, y) d z d s \\
& \approx \int_{0}^{1 / 2} \int_{B(0,3)} p(s, x, z) \frac{1}{|z|^{\alpha}} p(1, x, y) d z d s \\
& \approx \int_{0}^{1 / 2} \int_{\mathbb{R}^{d}} p(s, x, z) \frac{1}{|z|^{\alpha}} p(1, x, y) d z d s \\
& =G(1 / 2, x) p(1, x, y) \approx G(1, x) p(1, x, y),
\end{aligned}
$$

where in the second step we used the fact that

$$
p(1-s, z, y) \approx p(1, x, y), \quad 0<s \leq 1 / 2,|x|<2,|z| \leq 3,|y|>4 .
$$


Next, suppose that $|x| \leq 1$ and $|x| \leq|y| \leq 4$. Then, $p(1, x, y) \approx c$. Note that

$$
\begin{aligned}
\int_{\mathbb{R}^{d}} p_{1}(t, x, z) p(r, z, y) d z & =\int_{\mathbb{R}^{d}} \int_{0}^{t} \int_{\mathbb{R}^{d}} p(s, x, w)|w|^{-\alpha} p(t-s, w, z) p(r, z, y) d w d s d z \\
& =\int_{0}^{t} \int_{\mathbb{R}^{d}} p(s, x, w)|w|^{-\alpha} p(t+r-s, w, y) d w d s \\
& \leq p_{1}(t+r, x, y) .
\end{aligned}
$$

Hence, by the scaling property of $p_{1}(t, x, y)$ and Eq. 3.12,

$$
\begin{aligned}
p_{1}(1, x, y) & \geq \int_{B(0,8)^{c}} p_{1}\left(1 / 2^{\alpha}, x, z\right) p\left(1-1 / 2^{\alpha}, z, y\right) d z \\
& =\int_{B(0,8)^{c}} 2^{d} p_{1}(1,2 x, 2 z) p\left(1-1 / 2^{\alpha}, z, y\right) d z \\
& \geq c_{3} G(1,2 x) \int_{B(0,8)^{c}} p(1,2 x, 2 z) p\left(1-1 / 2^{\alpha}, z, y\right) d z \\
& \geq c_{4} G(1, x) \geq c_{5} G(1, x) p(1, x, y),
\end{aligned}
$$

where in the third inequality we used the fact that

$$
\begin{aligned}
\int_{B(0,8)^{c}} p(1,2 x, 2 z) p\left(1-1 / 2^{\alpha}, z, y\right) d z & \geq c_{6} \int_{B(0,8)^{c}} \frac{1}{|z-x|^{d+\alpha}|z-y|^{d+\alpha}} d z \\
& \geq c_{7} \int_{B(0,8)^{c}} \frac{1}{|z|^{2 d+2 \alpha}} d z \geq c_{8} .
\end{aligned}
$$

At last, suppose that $1 \leq|x| \leq|y|$. Then $G(1, x) \approx|x|^{-\alpha}$. Hence,

$$
\begin{aligned}
p_{1}(1, x, y) & \geq \int_{0}^{1 / 2} \int_{B(|x|, 1 / 2)} p(s, x, z) \frac{1}{|z|^{\alpha}} p(1-s, z, y) d z d s \\
& \geq c_{9} \int_{0}^{1 / 2} \int_{B(|x|, 1 / 2)} p(s, x, z) \frac{1}{|x|^{\alpha}} p(1-s, z, y) d z d s \\
& \geq c_{10} G(1, x) p(1, x, y),
\end{aligned}
$$

where the last inequality follows from the facts that

$$
p(1-s, z, y) \approx p(1, x, y), \quad 0<s<1 / 2, z \in B(x, 1 / 2), 1 \leq|x| \leq|y|
$$

and

$$
\int_{0}^{1 / 2} \int_{B(|x|, 1 / 2)} p(s, x, z) d z d s \geq c_{11} .
$$

The proof is complete.

The following estimate is generally well known (see e.g. [9, Section 6] for further background).

Lemma 3.8 For all $t>0$ and $x, y \in \mathbb{R}_{0}^{d}$, we have

$$
\tilde{p}(t, x, y) \geq p(t, x, y) \exp \left[\kappa \frac{p_{1}(t, x, y)}{p(t, x, y)}\right] .
$$

Proof Since the proof is a little long, we will postpone it to the Appendix. 
We note that the estimate in Lemma 3.8 is not sharp. More precisely, one may show that $\lim _{s \rightarrow 0^{+}} \frac{p_{1}(1, s x, y)}{-\log (s|x|) p(1, s x, y)}=\frac{\Gamma\left(\frac{d-\alpha}{2}\right)}{2^{\alpha-1} \Gamma\left(\frac{\alpha}{2}\right) \Gamma\left(\frac{d}{2}\right)}$. Hence, by Eq. 1.3, for fixed $y \neq 0$, $\tilde{p}(1, x, y) e^{-\kappa \frac{p_{1}(1, x, y)}{p(1, x, y)}} \rightarrow \infty$ as $x \rightarrow 0$. However, we still can get the following useful estimate.

Corollary 3.9 There are constants $c, \gamma>0$ such that for all $t>0$ and $x, y \in \mathbb{R}_{0}^{d}$, we have

$$
\tilde{p}(t, x, y) \geq c\left[1 \wedge\left(t^{-1 / \alpha}|x|\right)^{\gamma}\right]\left[1 \wedge\left(t^{-1 / \alpha}|y|\right)^{\gamma}\right] p(t, x, y)
$$

Proof Lemmas 3.7 and 3.8 along with Eq. 3.11 yield that for any $x, y \in \mathbb{R}_{0}^{d}$,

$$
\begin{aligned}
\tilde{p}(t, x, y) & \geq p(t, x, y) \exp \left[\kappa \frac{p_{1}(t, x, y)}{p(t, x, y)}\right] \\
& \geq p(t, x, y) \exp [-c(G(t, x)+G(t, y))] \\
& \geq p(t, x, y) \exp \left[-C\left(\log \left(1+t|x|^{-\alpha}\right)+\log \left(1+t|y|^{-\alpha}\right)\right)\right] \\
& =p(t, x, y)\left[\left(1+t|x|^{-\alpha}\right)^{-C}\right]\left[\left(1+t|y|^{-\alpha}\right)^{-C}\right] \\
& \geq p(t, x, y)\left[2^{-C}\left(1 \vee t|x|^{-\alpha}\right)^{-C}\right]\left[2^{-C}\left(1 \vee t|y|^{-\alpha}\right)^{-C}\right],
\end{aligned}
$$

thus we get Eq. 3.13 with $\gamma=\alpha C$ and $c=4^{-C}$.

Lemma 3.10 For any $r>0$, there is a constant $C_{r}>0$ such that for all $x, y \in \mathbb{R}^{d}$ with $|x| \wedge|y| \geq r$

$$
\tilde{p}(1, x, y) \geq C_{r} p(1, x, y) \text {. }
$$

Proof For $r>0$ and $x, y \in \mathbb{R}^{d}$ with $|x| \wedge|y| \geq r$, by Eq. 3.13, we get

$$
\tilde{p}(1, x, y) \geq c(1 \wedge r)^{2 \gamma} p(1, x, y),
$$

where $c$ and $\gamma$ are the constants from Corollary 3.9.

Lemma 3.11 For any $R>0$, there is a constant $C_{R}>0$ such that for any $x, y \in \mathbb{R}^{d}$ with $|x| \vee|y| \leq R$

$$
\tilde{p}(1, x, y) \geq C_{R}|x|^{\delta}|y|^{\delta}
$$

Proof By Eq. 3.9, there exists a constant $R_{0} \geq 2 \cdot 3^{1 / \alpha}$ large enough such that for all $x \in B\left(0, R_{0} / 2\right)$,

$$
\int_{B\left(0, R_{0}\right)} \tilde{p}(1, x, y) d y \geq R_{0}^{-\delta} \int_{B\left(0, R_{0}\right)} \tilde{p}(1, x, y)|y|^{\delta} d y \geq \frac{|x|^{\delta}}{2 R_{0}^{\delta}} .
$$

On the other hand, by Eq. 3.5, for all $r_{0}>0$ and $x \in \mathbb{R}^{d}$,

$$
\int_{B\left(0, r_{0}\right)} \tilde{p}(1, x, y) d y \leq C_{1}|x|^{\delta} \int_{B\left(0, r_{0}\right)} p(1, x, y)|y|^{\delta} d y \leq C_{1}|x|^{\delta} r_{0}^{\delta} .
$$


We take $r_{0}=\frac{1}{\left(4 C_{1}\right)^{1 / \delta} R_{0}}$. For $0<a<b<\infty$, let $D(a, b)=B(0, b) \backslash B(0, a)$. Then, for $|x|<R_{0} / 2$,

$$
\int_{D\left(r_{0}, R_{0}\right)} \tilde{p}(1, x, z) d z \geq|x|^{\delta}\left(\frac{1}{2 R_{0}^{\delta}}-C_{1} r_{0}^{\delta}\right)=\frac{|x|^{\delta}}{4 R_{0}^{\delta}} .
$$

Therefore, by Eqs. 2.5 and 3.14, for all $x, y \in \mathbb{R}^{d}$ with $|x| \vee|y| \leq R_{0} / 2$,

$$
\begin{aligned}
\tilde{p}(3, x, y) & \geq \int_{D\left(r_{0}, R_{0}\right)} \int_{D\left(r_{0}, R_{0}\right)} \tilde{p}(1, x, z) \tilde{p}(1, z, w) \tilde{p}(1, w, y) d z d w \\
& \geq \frac{|x|^{\delta}|y|^{\delta}}{16 R_{0}^{2 \delta}} \inf _{z, w \in D\left(r_{0}, R_{0}\right)} \tilde{p}(1, z, w) .
\end{aligned}
$$

Next, by Lemma 3.10,

$$
\inf _{z, w \in D\left(r_{0}, R_{0}\right)} \tilde{p}(1, z, w) \geq C_{r_{0}} \inf _{z, w \in D\left(r_{0}, R_{0}\right)} p(1, z, w) \geq \frac{c C_{r_{0}}}{\left(2 R_{0}\right)^{d+\alpha}}>0,
$$

where $C_{r_{0}}>0$ is a constant given in Lemma 3.10. Hence,

$$
\tilde{p}(3, x, y) \geq c_{0}|x|^{\delta}|y|^{\delta}, \quad|x| \vee|y|<R_{0} / 2 .
$$

Now, by the scaling property of $\tilde{p}$, we obtain

$$
\tilde{p}(1, x, y)=3^{-d / \alpha} \tilde{p}\left(3,3^{-1 / \alpha} x, 3^{-1 / \alpha} y\right) \geq c_{0} 3^{-(d+2 \delta) / \alpha}|x|^{\delta}|y|^{\delta}, \quad|x| \vee|y| \leq \frac{R_{0}}{2 \cdot 3^{1 / \alpha}}
$$

This completes the proof.

Remark 3.12 Instead of applying Lemma 3.10, we can make use of the Feynman-Kac formula (2.6) for the semigroup $\left(\tilde{P}_{t}\right)_{t \geq 0}$ and Dirichlet heat kernel estimates for fractional Laplacian obtained in [14] to achieve (3.15).

Theorem 3.13 (Lower bounds) There is a constant $C>0$ such that for all $x, y \in \mathbb{R}^{d}$,

$$
\tilde{p}(1, x, y) \geq C\left(1 \wedge|x|^{\delta}\right)\left(1 \wedge|y|^{\delta}\right) p(1, x, y) .
$$

Proof By symmetry, we will consider only $|x| \leq|y|$. For $\left|w_{1}\right| \leq 1 / 4,\left|w_{2}\right|>1$ and $1 / 4 \leq|z| \leq 1 / 2$, by Lemmas 3.10 and 3.11, we have

$$
\tilde{p}\left(1, w_{1}, z\right) \geq c_{1}\left|w_{1}\right|^{\delta}
$$

and

$$
\tilde{p}\left(1, w_{2}, z\right) \geq c_{2} p\left(1, w_{2}, z\right) \approx p\left(2, w_{2}, z\right) \approx p\left(2, w_{2}, w_{1}\right) .
$$

Hence, for any $\left|w_{1}\right| \leq 1 / 4$ and $\left|w_{2}\right|>1$,

$$
\tilde{p}\left(2, w_{1}, w_{2}\right) \geq \int_{B(0,1 / 2) \backslash B(0,1 / 4)} \tilde{p}\left(1, w_{1}, z\right) \tilde{p}\left(1, z, w_{2}\right) d z \geq c\left|w_{1}\right|^{\delta} p\left(2, w_{1}, w_{2}\right) .
$$

Therefore, for $|x| \leq 2^{-1 / \alpha} / 4$ and $|y|>2^{-1 / \alpha}$, by Eq. 3.16,

$$
\begin{aligned}
\tilde{p}(1, x, y) & =2^{d / \alpha} \tilde{p}\left(2,2^{1 / \alpha} x, 2^{1 / \alpha} y\right) \geq c 2^{d / \alpha}\left|2^{1 / \alpha} x\right|^{\delta} p\left(2,2^{1 / \alpha} x, 2^{1 / \alpha} y\right) \\
& =c 2^{\delta / \alpha}|x|^{\delta} p(1, x, y) .
\end{aligned}
$$

Next, for $|x| \wedge|y| \geq 2^{-1 / \alpha} / 4$, we use Lemma 3.10. Finally, for $|x| \vee|y| \leq 2^{-1 / \alpha}$, we apply Lemma 3.11 . 
Two-sided estimates for $\tilde{p}(t, x, y)$ stated in Theorem 1.1 is a direct consequence of the scaling property of $\tilde{p}(t, x, y)$ and Theorems 3.5 and 3.13.

\subsection{Joint Continuity of $\tilde{p}(t, x, y)$}

To prove the joint continuity of $\tilde{p}(t, x, y)$, we just follow the same argument of [8, Subsection 4.3]. For the sake of completeness, we present the proof here.

Lemma 3.14 For any fixed $x \in \mathbb{R}_{0}^{d}$, the function $\mathbb{R}_{0}^{d} \ni y \mapsto \tilde{p}(t, x, y)$ is continuous.

Proof Fix $x, y, z \in \mathbb{R}_{0}^{d}$ with $z \rightarrow y$. Then, by Eq. 2.7,

$$
\begin{aligned}
\tilde{p}(1, x, y)-\tilde{p}(1, x, z)= & p(1, x, y)-p(1, x, z) \\
& +\int_{0}^{1} \int_{\mathbb{R}^{d}} \tilde{p}(1-s, x, w) q(w)(p(s, w, y)-p(s, w, z)) d w d s .
\end{aligned}
$$

For any $\varepsilon>0$ small enough, by Eq. 3.11,

$$
\begin{aligned}
& -\int_{0}^{\varepsilon} \int_{\mathbb{R}^{d}} \tilde{p}(1-s, x, w) q(w) p(s, w, y) d w d s \\
\leq & -\int_{0}^{\varepsilon} \int_{\mathbb{R}^{d}} p(1-s, x, w) q(w) p(s, w, y) d w d s \\
\leq & -c_{1} \int_{0}^{\varepsilon} \int_{\mathbb{R}^{d}} p(s, w, y) q(w) d w d s \\
= & -\kappa c_{1} G(\varepsilon, y) \leq c_{2} \varepsilon|y|^{-\alpha} .
\end{aligned}
$$

Similarly, we have

$$
-\int_{0}^{\varepsilon} \int_{\mathbb{R}^{d}} \tilde{p}(1-s, x, w) q(w) p(s, w, z) d w d s \leq c_{2} \varepsilon|z|^{-\alpha} .
$$

For any $\varepsilon \leq s \leq 1$ and $w, y, z \in \mathbb{R}^{d}$ with $z \rightarrow y$, we have $p(s, w, y) \asymp p(s, w, z)$. By the dominated convergence theorem, it holds that

$$
\int_{\varepsilon}^{1} \int_{\mathbb{R}^{d}} \tilde{p}(1-s, x, w) q(w)(p(s, w, y)-p(s, w, z)) d w d s \rightarrow 0, \quad z \rightarrow y .
$$

Combining with all the estimates above, we prove the desired assertion.

Proposition 3.15 The function $\tilde{p}(t, x, y)$ is jointly continuous with respect to $t>0$ and $x, y \in \mathbb{R}_{0}^{d}$.

Proof By the scaling property of $\tilde{p}(t, x, y)$, it suffices to show the continuity of $\tilde{p}(1, x, y)$ with respect to $x, y \in \mathbb{R}_{0}^{d}$. As indicated in the proof of Lemma 3.14, we only need to verify that

$$
-\int_{0}^{1} \int_{\mathbb{R}^{d}}|\tilde{p}(1-s, \tilde{x}, w) p(s, w, \tilde{y})-\tilde{p}(1-s, x, w) p(s, w, y)| q(w) d w d s \rightarrow 0
$$

for any $x, y, \tilde{x}, \tilde{y} \in \mathbb{R}_{0}^{d}$ with $\tilde{x} \rightarrow x$ and $\tilde{y} \rightarrow y$. 
In addition to Eq. 3.17, we have

$$
\begin{aligned}
& -\int_{1-\varepsilon}^{1} \int_{\mathbb{R}^{d}} \tilde{p}(1-s, x, w) q(w) p(s, w, y) d w d s \\
= & -\int_{0}^{\varepsilon} \int_{\mathbb{R}^{d}} \tilde{p}(s, x, w) q(w) p(1-s, w, y) d w d s \\
\leq & -\int_{0}^{\varepsilon} \int_{\mathbb{R}^{d}} p(s, x, w) q(w) p(1-s, w, y) d w d s \\
\leq & c_{1} \varepsilon|x|^{-\alpha} .
\end{aligned}
$$

For any $\varepsilon<s<1-\varepsilon$ and $x, y, z, \tilde{x}, \tilde{y} \in \mathbb{R}_{0}^{d}$ with $x \rightarrow \tilde{x}$ and $y \rightarrow \tilde{y}$, $p(s, z, \tilde{y}) \approx p(s, z, y)$, and $\tilde{p}(1-s, \tilde{x}, z) \approx p(1-s, x, z)$, thanks to Lemma 3.14. Then, by the dominated convergence theorem, it holds that

$$
-\int_{\varepsilon}^{1-\varepsilon} \int_{\mathbb{R}^{d}}|\tilde{p}(1-s, \tilde{x}, w) p(s, w, \tilde{y})-\tilde{p}(1-s, x, w) p(s, w, y)| q(w) d w d s \rightarrow 0
$$

for any $x, y, \tilde{x}, \tilde{y} \in \mathbb{R}_{0}^{d}$ with $\tilde{x} \rightarrow x$ and $\tilde{y} \rightarrow y$.

Hence, according to all the estimates above, we prove the desired assertion.

Theorem 3.16 (Joint continuity) The function $\tilde{p}(t, x, y)$ is jointly continuous with respect to $t>0$ and $x, y \in \mathbb{R}^{d}$.

Proof According to Proposition 3.15 and the scaling property of $\tilde{p}(t, x, y)$, we only need to verify that $p(1, x, y)$ is jointly continuous with respect to $x, y \in \mathbb{R}^{d}$ when $x=0$ or $y=0$. Since $\tilde{p}(1, x, y)=0$ when $x=0$ or $y=0$, the desired assertion for the joint continuity is a direct consequence of the fact that $\tilde{p}(1, x, y) \geq 0$ and two-sided estimates for $\tilde{p}(1, x, y)$ on $\mathbb{R}_{0}^{d} \times \mathbb{R}_{0}^{d}$.

\subsection{Dirichlet Forms}

Finally, we discuss the Dirichlet form associated with the Schrödinger operator $\mathcal{L}$ given by Eq. 1.1; see [21] for the theory of Dirichlet forms. According to [19, Theorem 2.5], the Feynman-Kac semigroup $\left(\tilde{P}_{t}\right)_{t \geq 0}$ in $L^{2}\left(\mathbb{R}_{0}^{d} ; d x\right)$ coincides with the semigroup corresponding to $\tilde{\mathscr{E}}$ with the domain

$$
\mathscr{D}(\tilde{\mathscr{E}})=\left\{f \in L^{2}\left(\mathbb{R}_{0}^{d} ; d x\right): \iint_{\mathbb{R}_{0}^{d} \times \mathbb{R}_{0}^{d}} \frac{(f(x)-f(y))^{2}}{|x-y|^{d+\alpha}} d x d y+\int_{\mathbb{R}_{0}^{d}} f^{2}(x)|q(x)| d x<\infty\right\}
$$

and defined by

$$
\begin{aligned}
\tilde{E}(f, g)= & \frac{1}{2} \iint_{\mathbb{R}_{0}^{d} \times \mathbb{R}_{0}^{d}}(f(x)-f(y))(g(x)-g(y)) v(x-y) d x d y \\
& +\int_{\mathbb{R}_{0}^{d}} f(x) g(x)|q(x)| d x
\end{aligned}
$$


for any $f, g \in \mathscr{D}(\tilde{\mathscr{E}})$, where $v$ is defined by Eq. 2.1. Clearly, the quadratic form $(\tilde{\mathscr{E}}, \mathscr{D}(\tilde{\mathscr{E}}))$ is equivalently given by

$$
\begin{aligned}
\tilde{\mathscr{E}}(f, g)= & \frac{1}{2} \iint_{\mathbb{R}^{d} \times \mathbb{R}^{d}}(f(x)-f(y))(g(x)-g(y)) v(x-y) d x d y \\
& +\int_{\mathbb{R}^{d}} f(x) g(x)|q(x)| d x, \\
\mathscr{D}(\tilde{\mathscr{E}})= & \left\{f \in L^{2}\left(\mathbb{R}^{d} ; d x\right): \tilde{\mathscr{E}}(f, f)<\infty\right\},
\end{aligned}
$$

which are extended to be defined on $L^{2}\left(\mathbb{R}^{d} ; d x\right)$.

Proposition $3.17(\tilde{\mathscr{E}}, \mathscr{D}(\tilde{\mathscr{E}}))$ is a symmetric regular Dirichlet form on $L^{2}\left(\mathbb{R}^{d} ; d x\right)$ with core $C_{c}^{\infty}\left(\mathbb{R}^{d}\right)$.

\section{Proof Define}

$$
\mathscr{E}(f, g)=\frac{1}{2} \iint_{\mathbb{R}^{d} \times \mathbb{R}^{d}}(f(x)-f(y))(g(x)-g(y)) v(x-y) d x d y, \quad f, g \in \mathscr{D}(\mathscr{E})
$$

and

$$
\mathscr{D}(\mathscr{E})=\left\{f \in L^{2}\left(\mathbb{R}^{d} ; d x\right): \mathscr{E}(f, f)<\infty\right\} .
$$

Then, $(\mathscr{E}, \mathscr{D}(\mathscr{E}))$ is a symmetric Dirichlet form on $L^{2}\left(\mathbb{R}^{d} ; d x\right)$ associated with fractional Laplacian; moreover, $C_{c}^{\infty}\left(\mathbb{R}^{d}\right) \subset \mathscr{D}\left(\Delta^{\alpha / 2}\right)$ (here $\mathscr{D}\left(\Delta^{\alpha / 2}\right)$ denotes the $L^{2}$-domain of $\Delta^{\alpha / 2}$ on $\left.L^{2}\left(\mathbb{R}^{d} ; d x\right)\right)$ and $(\mathscr{E}, \mathscr{D}(\mathscr{E}))$ is regular with core $C_{c}^{\infty}\left(\mathbb{R}^{d}\right)$; see [13, Section 2.2.2] for more details. On the other hand, due to $\alpha<d$, we can verify that

$$
\int_{\mathbb{R}^{d}} f(x)^{2}|q(x)| d x<\infty, \quad f \in C_{c}^{\infty}\left(\mathbb{R}^{d}\right) .
$$

In particular, $C_{c}^{\infty}\left(\mathbb{R}^{d}\right) \subset \mathscr{D}\left(\Delta^{\alpha / 2}\right) \cap L^{2}\left(\mathbb{R}^{d} ;|q(x)| d x\right) \subset \mathscr{D}(\tilde{\mathscr{E}})$.

It is easy to prove that $(\tilde{\mathscr{E}}, \mathscr{D}(\tilde{\mathscr{E}}))$ is a symmetric Dirichlet form on $L^{2}\left(\mathbb{R}^{d} ; d x\right)$. Next, we claim that $C_{c}^{\infty}\left(\mathbb{R}^{d}\right)$ is dense in $\mathscr{D}(\tilde{\mathscr{E}})$ with the norm $\sqrt{\tilde{\mathscr{E}}}+\|\cdot\|_{L^{2}\left(\mathbb{R}^{d} ; d x\right)}$. According to the Hardy inequality for fractional Laplacian (see [7, Proposition 5]), there is a constant $C_{0}>0$ such that for all $f \in L^{2}\left(\mathbb{R}^{d} ; d x\right)$,

$$
\int_{\mathbb{R}^{d}} f^{2}(x)|q(x)| d x \leq C_{0} \mathscr{E}(f, f) .
$$

Thus, the norms $\sqrt{\tilde{\mathscr{E}}}+\|\cdot\|_{L^{2}\left(\mathbb{R}^{d} ; d x\right)}$ and $\sqrt{\mathscr{E}}+\|\cdot\|_{L^{2}\left(\mathbb{R}^{d} ; d x\right)}$ are equivalent. Therefore, the desired assertion above immediately follows from the fact that $(\mathscr{E}, \mathscr{D}(\mathscr{E}))$ is regular with core $C_{c}^{\infty}\left(\mathbb{R}^{d}\right)$.

Let $h(x)=|x|^{\delta}$, and define

$$
\overline{\mathscr{E}}(f, f)=\frac{1}{2} \iint_{\mathbb{R}^{d} \times \mathbb{R}^{d}}\left(\frac{f(x)}{h(x)}-\frac{f(y)}{h(y)}\right)^{2} h(x) h(y) v(x-y) d x d y, \quad f \in \mathscr{D}(\overline{\mathscr{E}}),
$$

where $\mathscr{D}(\overline{\mathscr{E}})=\left\{f \in L^{2}\left(\mathbb{R}^{d} ; d x\right): \overline{\mathscr{E}}(f, f)<\infty\right\}$.

Proposition 3.18 We have $\mathscr{D}(\tilde{\mathscr{E}})=\mathscr{D}(\overline{\mathscr{E}})$ and

$$
\tilde{\mathscr{E}}(f, f)=\overline{\mathscr{E}}(f, f), \quad f \in \mathscr{D}(\tilde{\mathscr{E}}) .
$$


Proof Denote by $\langle\cdot, \cdot\rangle$ the inner product of $L^{2}\left(\mathbb{R}^{d} ; d x\right)$. Recall that $\left(\tilde{P}_{t}\right)_{t \geq 0}$ is well defined on $L^{2}\left(\mathbb{R}^{d} ; d x\right)$ by setting $\tilde{P}_{t} f(0)=0$ for any $f \in L^{2}\left(\mathbb{R}^{d} ; d x\right)$. According to Eq. 2.16,

$$
\begin{aligned}
\left\langle f-\tilde{P}_{t} f, f\right\rangle & =\int_{\mathbb{R}^{d}}\left(f(x)-\int_{\mathbb{R}^{d}} \tilde{p}(t, x, y) f(y) d y\right) f(x) d x \\
& =\int_{\mathbb{R}^{d}}\left(\frac{f(x)}{h(x)} \int_{\mathbb{R}^{d}} \tilde{p}(t, x, y) h(y) d y-\int_{\mathbb{R}^{d}} \tilde{p}(t, x, y) h(y) \frac{f(y)}{h(y)} d y\right) f(x) d x \\
& =\iint_{\mathbb{R}^{d} \times \mathbb{R}^{d}} \tilde{p}(t, x, y)\left(\frac{f(x)}{h(x)}-\frac{f(y)}{h(y)}\right) \frac{f(x)}{h(x)} h(x) h(y) d x d y .
\end{aligned}
$$

Hence, by the symmetry,

$$
\left\langle f-\tilde{P}_{t} f, f\right\rangle=\frac{1}{2} \iint_{\mathbb{R}^{d} \times \mathbb{R}^{d}} \tilde{p}(t, x, y)\left(\frac{f(x)}{h(x)}-\frac{f(y)}{h(y)}\right)^{2} h(x) h(y) d x d y .
$$

Note that

$$
\lim _{t \rightarrow 0^{+}} \frac{\tilde{p}(t, x, y)}{t}=v(x-y), \quad x, y \in \mathbb{R}_{0}^{d} .
$$

Indeed, by the Duhamel formula (2.7), for any $x, y \in \mathbb{R}_{0}^{d}$,

$$
\begin{aligned}
\lim _{t \rightarrow 0^{+}} \frac{\tilde{p}(t, x, y)}{t} & =\lim _{t \rightarrow 0^{+}} \frac{p(t, x, y)}{t}+\lim _{t \rightarrow 0^{+}} \frac{1}{t} \int_{0}^{t} \int_{\mathbb{R}^{d}} \tilde{p}(t-s, x, z) q(z) p(s, z, y) d z d s \\
& =v(x-y)+\lim _{t \rightarrow 0^{+}} \frac{1}{t} \int_{0}^{t} \int_{\mathbb{R}^{d}} \tilde{p}(t-s, x, z) q(z) p(s, z, y) d z d s .
\end{aligned}
$$

Next, by the fact that $\tilde{p}(t, x, y) \leq p(t, x, y)$ for all $t>0$ and $x, y \in \mathbb{R}^{d}$, Lemma 3.7, Eqs. 3.10 and 3.11, for any $x, y \in \mathbb{R}_{0}^{d}$,

$\frac{1}{t} \int_{0}^{t} \int_{\mathbb{R}^{d}} \tilde{p}(t-s, x, z)|z|^{-\alpha} p(s, z, y) d z d s \leq c_{1} t^{-1} p(t, x, y)[G(t, x)+G(t, y)] \stackrel{t \rightarrow 0}{\longrightarrow} 0$,

and so we get Eq. 3.18 .

Now, for $f \in \mathscr{D}(\tilde{\mathscr{E}})$, by Fatou's Lemma and Eq. 3.18, we have

$$
\begin{aligned}
\tilde{\mathscr{E}}(f, f) & =\lim _{t \rightarrow 0} \frac{1}{t}\left\langle f-\tilde{P}_{t} f, f\right\rangle \\
& =\lim _{t \rightarrow 0} \frac{1}{2} \iint_{\mathbb{R}^{d} \times \mathbb{R}^{d}} \frac{\tilde{p}(t, x, y)}{t}\left(\frac{f(x)}{h(x)}-\frac{f(y)}{h(y)}\right)^{2} h(x) h(y) d y d x \\
& \geq \frac{1}{2} \iint_{\mathbb{R}_{0}^{d} \times \mathbb{R}_{0}^{d}} \liminf _{t \rightarrow 0} \frac{\tilde{p}(t, x, y)}{t}\left(\frac{f(x)}{h(x)}-\frac{f(y)}{h(y)}\right)^{2} h(x) h(y) d y d x \\
& =\frac{1}{2} \iint_{\mathbb{R}_{0}^{d} \times \mathbb{R}_{0}^{d}}\left(\frac{f(x)}{h(x)}-\frac{f(y)}{h(y)}\right)^{2} h(x) h(y) v(x-y) d y d x=\overline{\mathscr{E}}(f, f) .
\end{aligned}
$$


Hence, $\mathscr{D}(\tilde{\mathscr{E}}) \subset \mathscr{D}(\overline{\mathscr{E}})$. On the other hand, we take $f \in \mathscr{D}(\overline{\mathscr{E}})$. Since $\tilde{p}(t, x, y) \leq$ $p(t, x, y) \leq c_{2} t v(x-y)$ for all $t>0$ and $x, y \in \mathbb{R}^{d}$, according to the dominated convergence theorem and Eq. 3.18 again, we have

$$
\begin{aligned}
\tilde{E}(f, f) & =\lim _{t \rightarrow 0} \frac{1}{t}\left\langle f-\tilde{P}_{t} f, f\right\rangle \\
& =\lim _{t \rightarrow 0} \frac{1}{2} \iint_{\mathbb{R}^{d} \times \mathbb{R}^{d}} \frac{\tilde{p}(t, x, y)}{t}\left(\frac{f(x)}{h(x)}-\frac{f(y)}{h(y)}\right)^{2} h(x) h(y) d y d x \\
& =\frac{1}{2} \iint_{\mathbb{R}^{d} \times \mathbb{R}^{d}}\left(\frac{f(x)}{h(x)}-\frac{f(y)}{h(y)}\right)^{2} h(x) h(y) v(x-y) d y d x=\overline{\mathscr{E}}(f, f) .
\end{aligned}
$$

Combining with both inequalities, we prove the desired assertion.

Remark 3.19 The construction of $(\overline{\mathscr{E}}, \mathscr{D}(\overline{\mathscr{E}}))$ can be deduced from Doob's theory of $h$ transformations; see [18, Chapter 11] for more details. Indeed, as shown by Eq. 2.14, the function $h=|x|^{\delta}$ is harmonic with respect to the operator $\mathcal{L}$ given by Eq. 1.1. Define $\mathcal{L}_{h} f(x):=h(x)^{-1} \mathcal{L}(f h)(x)$ for all $f \in L^{2}\left(\mathbb{R}^{d} ; h(x)^{2} d x\right)$. It is easy to see that the operator $\mathcal{L}_{h}$ is symmetric on $L^{2}\left(\mathbb{R}^{d} ; h(x)^{2} d x\right)$, and the associated symmetric regular Dirichlet form $\left(\mathscr{E}_{h}, \mathscr{D}\left(\mathscr{E}_{h}\right)\right)$ on $L^{2}\left(\mathbb{R}^{d} ; h(x)^{2} d x\right)$ is given by

$$
\begin{aligned}
\mathscr{E}_{h}(f, f)= & -\left\langle\mathcal{L}_{h} f, f\right\rangle_{L^{2}\left(\mathbb{R}^{d} ; h(x)^{2} d x\right)}=-\iint_{\mathbb{R}^{d} \times \mathbb{R}^{d}}(f(y) h(y)-f(x) h(x)) f(x) h(x) v(x-y) d y d x \\
& -\kappa \int_{\mathbb{R}^{d}}|x|^{-\alpha} f(x)^{2} h^{2}(x) d x \\
= & -\iint_{\mathbb{R}^{d} \times \mathbb{R}^{d}}(f(y)-f(x)) f(x) h(y) h(x) v(x-y) d y d x \\
& -\iint_{\mathbb{R}^{d} \times \mathbb{R}^{d}}(h(y)-h(x)) f(x)^{2} h(x) v(x-y) d y d x \\
& -\kappa \int_{\mathbb{R}^{d}}|x|^{-\alpha} f(x)^{2} h^{2}(x) d x \\
= & -\iint_{\mathbb{R}^{d} \times \mathbb{R}^{d}}(f(y)-f(x)) f(x) h(y) h(x) v(x-y) d y d x \\
= & \frac{1}{2} \iint_{\mathbb{R}^{d} \times \mathbb{R}^{d}}(f(y)-f(x))^{2} h(y) h(x) v(x-y) d y d x
\end{aligned}
$$

for all $f \in \mathscr{D}\left(\mathscr{E}_{h}\right)$, where in the fourth equality we used Eq. 2.14 and the last equality follows form the property that $v(x-y)=v(y-x)$. Note that

$$
\mathscr{E}_{h}(f, f)=-\left\langle\mathcal{L}_{h} f, f\right\rangle_{L^{2}\left(\mathbb{R}^{d} ; h(x)^{2} d x\right)}=-\langle\mathcal{L}(h f), h f\rangle_{L^{2}\left(\mathbb{R}^{d} ; d x\right)}=\tilde{\mathscr{E}}(h f, h f) .
$$

Combining both equalities above together, we arrive at

$$
\tilde{\mathscr{E}}(f, f)=\mathscr{E}_{h}\left(f h^{-1}, f h^{-1}\right)=\frac{1}{2} \iint_{\mathbb{R}^{d} \times \mathbb{R}^{d}}\left(\frac{f(y)}{h(y)}-\frac{f(x)}{h(x)}\right)^{2} h(y) h(x) v(x-y) d y d x .
$$

The right side of the equality above coincides with the expression of $(\overline{\mathscr{E}}, \mathscr{D}(\overline{\mathscr{E}}))$.

Acknowledgements We would like to thank Krzysztof Bogdan and Kamil Kaleta for interesting discussions and helpful comments. We also would like to thank the referee for his/her careful reading and numerous corrections. 
Open Access This article is distributed under the terms of the Creative Commons Attribution 4.0 International License (http://creativecommons.org/licenses/by/4.0/), which permits unrestricted use, distribution, and reproduction in any medium, provided you give appropriate credit to the original author(s) and the source, provide a link to the Creative Commons license, and indicate if changes were made.

\section{Appendix: Proof of Lemma 3.8}

Let $q_{0}(x)=-|x|^{-\alpha}$. For any $\lambda \geq 0$, denote by $p^{\lambda}(t, x, y)$ the heat kernel associated with the generator $\Delta^{\alpha / 2}+\lambda q_{0}(x)$. Hence, by Duhamel's formula (see [19, Propositions 5.2 and 5.3] and their proofs), we have

$$
p^{\lambda}(t, x, y)=p^{v}(t, x, y)+(\lambda-v) \int_{0}^{t} \int_{\mathbb{R}_{0}^{d}} p^{v}(t-s, x, z) q_{0}(z) p^{\lambda}(s, z, y) d z d s
$$

for any $t>0$ and $x, y \in \mathbb{R}_{0}^{d}$. Noting that $q_{0}(x)<0$ for all $\in \mathbb{R}_{0}^{d}$, we can rewrite the equality above as

$$
p^{v}(t, x, y)=p^{\lambda}(t, x, y)+\int_{0}^{t} \int_{\mathbb{R}_{0}^{d}} p^{v}(t-s, x, z)(\lambda-v)\left|q_{0}(z)\right| p^{\lambda}(s, z, y) d z d s .
$$

For any $\lambda \geq 0, t>0$ and $x, y \in \mathbb{R}_{0}^{d}$, we set

$$
\begin{aligned}
& p_{0}^{\lambda}(t, x, y)=p^{\lambda}(t, x, y), \\
& p_{n}^{\lambda}(t, x, y)=\int_{0}^{t} \int_{\mathbb{R}_{0}^{d}} p_{n-1}^{\lambda}(t-s, x, z)\left|q_{0}(z)\right| p^{\lambda}(s, z, y) d z d s, \quad n \geq 1 .
\end{aligned}
$$

Then, by [9, Lemma 1 and the proof of Theorem 2],

$$
p_{n+m+1}^{\lambda}(t, x, y)=\int_{0}^{t} \int_{\mathbb{R}_{0}^{d}} p_{n}^{\lambda}(t-s, x, z)\left|q_{0}(z)\right| p_{m}^{\lambda}(s, z, y) d z d s, \quad m, n \geq 0
$$

and

$$
p^{\eta-\lambda}(t, x, y)=\sum_{n=0}^{\infty} \lambda^{n} p_{n}^{\eta}(t, x, y), \quad \eta>\lambda>0
$$

Furthermore, we have

Lemma A.1 Let $0<\lambda<\eta<\infty$. For all $x, y \in \mathbb{R}_{0}^{d}$ and $t>0$,

$$
\sum_{n=k}^{\infty}\left(\begin{array}{l}
n \\
k
\end{array}\right) \lambda^{n-k} p_{n}^{\eta}(t, x, y)=p_{k}^{\eta-\lambda}(t, x, y), \quad k=0,1,2, \cdots
$$


Proof We use induction. When $k=0$, Eq. 1 holds trivially. For $k=1$,

$$
\begin{aligned}
p_{1}^{\eta-\lambda}(t, x, y) & =\int_{0}^{t} \int_{\mathbb{R}_{0}^{d}} p^{\eta-\lambda}(t-s, x, z)\left|q_{0}(z)\right| p^{\eta-\lambda}(s, z, y) d z d s \\
& =\int_{0}^{t} \int_{\mathbb{R}_{0}^{d}} \sum_{i=0}^{\infty} \lambda^{i} p_{i}^{\eta}(t-s, x, z)\left|q_{0}(z)\right| \sum_{j=0}^{\infty} \lambda^{j} p_{j}^{\eta}(s, z, y) d z d s \\
& =\sum_{i=0}^{\infty} \sum_{j=0}^{\infty} \lambda^{i+j} p_{i+j+1}^{\eta}(t, x, y)=\sum_{n=1}^{\infty} n \lambda^{n-1} p_{n}^{\eta}(t, x, y) .
\end{aligned}
$$

Next, we assume that Eq. 1 holds for some $k \in \mathbb{N}_{+}$. We get

$$
\begin{aligned}
p_{k+1}^{\eta-\lambda}(t, x, y) & =\int_{0}^{t} \int_{\mathbb{R}_{0}^{d}} p_{k}^{\eta-\lambda}(t-s, x, z)\left|q_{0}(z)\right| p^{\eta-\lambda}(s, z, y) d z d s \\
& =\int_{0}^{t} \int_{\mathbb{R}_{0}^{d}} \sum_{i=k}^{\infty}\left(\begin{array}{l}
i \\
k
\end{array}\right) \lambda^{i-k} p_{i}^{\eta}(t-s, x, z)\left|q_{0}(z)\right| \sum_{j=0}^{\infty} \lambda^{j} p_{j}^{\eta}(s, z, y) d z d s \\
& =\sum_{i=k}^{\infty} \sum_{j=0}^{\infty}\left(\begin{array}{l}
i \\
k
\end{array}\right) \lambda^{i+j-k} p_{i+j+1}^{\eta}(t, x, y) \\
& =\sum_{n=k+1}^{\infty} \sum_{j=0}^{n-k-1}\left(\begin{array}{c}
n-j-1 \\
k
\end{array}\right) \lambda^{n-k-1} p_{n}^{\eta}(t, x, y) \\
& =\sum_{n=k+1}^{\infty}\left(\begin{array}{c}
n \\
k+1
\end{array}\right) \lambda^{n-k-1} p_{n}^{\eta}(t, x, y),
\end{aligned}
$$

where in the last equality we used the fact proved in the proof of [9, Lemma 6] (cf. [22, (5.26)]). The proof is complete.

Next, we consider some properties of the function $\lambda \mapsto p^{\lambda}(t, x, y)$.

Lemma A.2 For fixed $x, y \in \mathbb{R}_{0}^{d}$ and $t>0$, the function

$$
h(\lambda)=p^{\lambda}(t, x, y), \quad \lambda>0
$$

is completely monotone, i.e., $(-1)^{k} h^{(k)}(\lambda) \geq 0$ for all $\lambda>0$ and $k=0,1,2, \cdots$.

Proof For $\lambda>0$, we take $\eta>\lambda$. Choosing $k=0$ in Eq. 1 , we get

$$
h(\lambda)=\sum_{n=0}^{\infty}(\eta-\lambda)^{n} p_{n}^{\eta}(t, x, y) \text {. }
$$

By Eq. 1, we get

$$
\frac{d^{k}}{d \lambda^{k}} h(\lambda)=k ! \sum_{n=k}^{\infty}(-1)^{k}\left(\begin{array}{l}
n \\
k
\end{array}\right)(\eta-\lambda)^{n-k} p_{n}^{\eta}(t, x, y)=(-1)^{k} k ! p_{k}^{\lambda}(t, x, y) .
$$

Since $p_{k}^{\lambda}(t, x, y) \geq 0$, we conclude that $h$ is completely monotone on $(0, \infty)$.

By the Bernstein theorem (see [32, Theorem 1.4]), we get 
Corollary A.3 For fixed $x, y \in \mathbb{R}_{0}^{d}$ and $t>0$, there exists a nonnegative Borel measure $\mu_{t, x, y}(d u)$ on $[0, \infty)$ such that

$$
p^{\lambda}(t, x, y)=\int_{0}^{\infty} e^{-\lambda u} \mu_{t, x, y}(d u) .
$$

The next lemma will yield the monotonicity of the function $\lambda \mapsto \frac{p_{1}^{\lambda}(t, x, y)}{p^{\lambda}(t, x, y)}$.

Lemma A.4 For every $n \geq 1, \lambda \geq 0, t>0$ and $x, y \in \mathbb{R}_{0}^{d}$, we have

$$
(n+1) p_{n+1}^{\lambda}(t, x, y) p_{n-1}^{\lambda}(t, x, y) \geq n p_{n}^{\lambda}(t, x, y)^{2} .
$$

Proof Fix $x, y \in \mathbb{R}_{0}^{d}$ and $t>0$, and let $\mu=\mu_{t, x, y}$ be the nonnegative measure from Corollary A.3. Then, by Eq. 2,

$$
n ! p_{n}^{\lambda}(t, x, y)=(-1)^{n} \frac{d^{n}}{d \lambda^{n}} p^{\lambda}(t, x, y)=\int_{0}^{\infty} e^{-\lambda u} u^{n} \mu(d u) .
$$

According to the Cauchy-Schwarz inequality,

$$
\begin{aligned}
{\left[\int_{0}^{\infty} e^{-\lambda u} u^{n} \mu(d u)\right]^{2} } & =\left[\int_{0}^{\infty} e^{-\lambda u / 2} u^{(n+1) / 2} \cdot e^{-\lambda u / 2} u^{(n-1) / 2} \mu(d u)\right]^{2} \\
& \leq\left(\int_{0}^{\infty} e^{-\lambda u} u^{n+1} \mu(d u)\right)\left(\int_{0}^{\infty} e^{-\lambda u} u^{n-1} \mu(d u)\right) .
\end{aligned}
$$

Hence,

$$
\left(n ! p_{n}^{\lambda}(t, x, y)\right)^{2} \leq(n+1) ! p_{n+1}^{\lambda}(t, x, y) \cdot(n-1) ! p_{n-1}^{\lambda}(t, x, y),
$$

and so the desired assertion follows.

Lemma A.5 For fixed $x, y \in \mathbb{R}_{0}^{d}$ and $t>0$, the function $\lambda \mapsto \frac{p_{1}^{\lambda}(t, x, y)}{p^{\lambda}(t, x, y)}$ is decreasing on $(0, \infty)$.

Proof Let $H(\lambda)=\frac{-h^{\prime}(\lambda)}{h(\lambda)}=\frac{p_{1}^{\lambda}(t, x, y)}{p^{\lambda}(t, x, y)}$ for $\lambda>0$, where in the second equality we used Eq. 2. Combining Eq. 2 again with Lemma A.4, we find that

$$
H^{\prime}(\lambda)=\frac{-h^{\prime \prime}(\lambda) h(\lambda)+h^{\prime}(\lambda)^{2}}{h^{2}(\lambda)}=\frac{-2 p_{2}^{\lambda}(t, x, y) p^{\lambda}(t, x, y)+p_{1}^{\lambda}(t, x, y)^{2}}{p^{\lambda}(t, x, y)^{2}} \leq 0,
$$

which yields the desired assertion.

We now present the main result in this appendix, which immediately gives us Lemma 3.8 .

Theorem A.6 For every $\lambda>0, t>0$ and $x, y \in \mathbb{R}_{0}^{d}$, we have

$$
p^{\lambda}(t, x, y) \geq p(t, x, y) \exp \left[\frac{\lambda p_{1}(t, x, y)}{p(t, x, y)}\right] \text {. }
$$


Proof Fix $x, y \in \mathbb{R}_{0}^{d}$ and $t>0$, and let $h(\lambda)=p^{\lambda}(t, x, y)$. Since $h \geq 0$,

$$
h(\lambda)=h(0) \exp \left[\int_{0}^{\lambda}(\log h(u))^{\prime} d u\right]=h(0) \exp \left[\int_{0}^{\lambda} \frac{h^{\prime}(u)}{h(u)} d u\right] .
$$

By Eq. 2 and Lemma A.5, we get

$$
p^{\lambda}(t, x, y)=p(t, x, y) \exp \left[-\int_{0}^{\lambda} \frac{p_{1}^{u}(t, x, y)}{p^{u}(t, x, y)} d u\right] \geq p(t, x, y) \exp \left[-\frac{\lambda p_{1}(t, x, y)}{p(t, x, y)}\right] \text {. }
$$

The proof is complete.

\section{References}

1. Abdellaoui, B., Medina, M., Peral, I., Primo, A.: The effect of the Hardy potential in some calderónZygmund properties for the fractional Laplacian. J. Differential Equations 260, 8160-8206 (2016)

2. Abdellaoui, B., Medina, M., Peral, I., Primo, A.: Optimal results for the fractional heat equation involving the Hardy potential. Nonlinear Anal. 140, 166-207 (2016)

3. Andrews, G.E., Askey, R., Roy, R.: Special functions. Cambridge University Press, Cambridge (1999)

4. Baras, P., Goldstein, J.A.: The heat equation with a singular potential. Trans. Amer. Math. Soc. 284, 121-139 (1984)

5. BenAmor, A.: The heat equation for the Dirichlet fractional Laplacian with Hardy's potentials: properties of minimal solutions and blow-up. arXiv:1606.01784

6. Bogdan, K., Byczkowski, T., Kulczycki, T., Ryznar, M., Song, R., Vondraček, Z.: Potential analysis of stable processes and its extensions, Lect. Notes Math, vol. 1980. Springer, Berlin (2009)

7. Bogdan, K., Dyda, B., Kim, P.: Hardy inequalities and non-explosion results for semigroups. Potential Anal. 44, 229-247 (2016)

8. Bogdan, K., Grzywny, T., Jakubowski, T., Pilarczyk, D.: Fractional Laplacian with Hardy potential. Comm. Partial Differential Equations 44, 20-50 (2019)

9. Bogdan, K., Hansen, W., Jakubowski, T.: Time-dependent Schrödinger perturbations of transition densities. Stud. Math. 189, 235-254 (2008)

10. Bogdan, K., Jakubowski, T.: Estimates of heat kernel of fractional Laplacian perturbed by gradient operators. Comm. Math. Phys. 271, 179-198 (2007)

11. Bogdan, K., Jakubowski, T.: Estimates of the Green function for the fractional Laplacian perturbed by gradient. Potential Anal. 36, 455-481 (2012)

12. Bogdan, K., Jakubowski, T., Sydor, S.: Estimates of perturbation series for kernels. J. Evol. Equ. 12, 973-984 (2012)

13. Chen, Z.-Q., Fukushima, M.: Symmetric Markov processes, time change, and boundary theory. Princeton University Press, Princeton (2012)

14. Chen, Z.-Q., Kim, P., Song, R.: Heat kernel estimates for Dirichlet fractional Laplacian. J. Eur. Math. Soc. 124, 1307-1329 (2010)

15. Chen, Z.-Q., Kim, P., Song, R.: Dirichlet heat kernel estimates for fractional Laplacian with gradient perturbation. Ann. Probab. 40, 2483-2538 (2012)

16. Chen, Z.-Q., Kim, P., Song, R.: Stability of Dirichlet heat kernel estimates for non-local operators under Feynman-Kac perturbation. Trans. Amer. Math. Soc. 367, 5237-5270 (2015)

17. Cho, S., Kim, P., Song, R., Vondraček, Z.: Factorization and estimates of Dirichlet heat kernels for non-local operators with critical killings. arXiv:1809.01782

18. Chung, K.L., Walsh, J.B.: Markov processes, Brownian motion, and time symmetry. Springer, Berlin (2004). 2nd

19. Demuth, M., van Casteren, J.A.: Stochastic spectral theory for selfadjoint Feller operators. A functional analysis approach. Basel, Birkhäuser Verleg (2000)

20. Frank, R.L., Lieb, E.H., Seiringer, R.: Hardy-lieb-thirring inequalities for fractional Schrödinger operators. J. Amer. Math. Soc. 21, 925-950 (2008)

21. Fukushima, M., Oshima, Y., Takeda, M.: Dirichlet forms and symmetric Markov processes. De Gruyter, Berlin (2011). 2nd

22. Graham, R.L., Kunth, D.E., Patashnik, O.: Concrete mathematics. Addison-Wesley, Reading (1994)

23. Ishige, K., Kabeya, Y., Ouhabaz, E.M.: The heat kernel of a Schrödinger operator with inverse sequare potential. Proc. London Math. Soc. 115, 381-410 (2017) 
24. Jakubowski, T.: On combinatorics of Schrödinger perturbations. Potential Anal. 31, 45-55 (2009)

25. Jakubowski, T., Serafin, G.: Stable estimates for source solution of critical fractal Burgers equation. Nonlinear Anal. 130, 396-407 (2016)

26. Kesten, H.: Hitting probabilities of single points for processes with stationary independent increments. In: Memoirs of the American Mathematical Society, Vol. 93, American Mathematical Society, Providence (1969)

27. Kim, D., Kuwae, K.: General analytic characterization of gaugeability for Feynman-Kac functionals. Math. Ann. 370, 1-37 (2018)

28. Kulczycki, T.: Gradient estimates of $q$-harmonic functions of fractional Schrödinger operator. Potential Anal. 39, 69-98 (2013)

29. Liskevich, V., Sobol, Z.: Estimates of integral kernels for semigroups associated with second-order elliptic operators with singular coefficients. Potential Anal. 18, 359-390 (2003)

30. Milman, P.D., Semenov, Y.A.: Global heat kernel bounds via desingularizing weights. J. Funct. Anal. 212, 373-398 (2004)

31. Milman, P.D., Semenov, Y.A.: Corrigendum to: Global heat kernel bounds via desingularizing weights [J. Funct. Anal., 212 (2004), 373-398]. J. Funct. Anal. 229, 238-239 (2005)

32. Schilling, R.L., Song, R., Vondraček, Z.: Bernstein functions: Theory and applications. Walter de Gruyter, Berlin (2010)

33. Song, R.: Two-sided estimates on the density of the Feynman-Kac semigroups of $\alpha$-stable-like processes. Electron. J. Probab. 11, 146-161 (2006)

34. Takeda, M.: Gaugeability for Feynman-Kac functionals with applications to symmetric $\alpha$-stable processes. Proc. Am. Math. Soc. 134, 2729-2738 (2006)

Publisher's Note Springer Nature remains neutral with regard to jurisdictional claims in published maps and institutional affiliations. 